\title{
Quantifying osmotic membrane fouling to enable comparisons across diverse processes
}

\author{
Emily W. Tow, John H. Lienhard V \\ Rohsenow Kendall Heat Transfer Laboratory \\ Department of Mechanical Engineering \\ Massachusetts Institute of Technology \\ Cambridge, Massachusetts 02139, USA
}

\begin{abstract}
In this study, a method of in situ membrane fouling quantification is developed that enables comparisons of foulant accumulation between desalination processes with different membranes, driving forces, and feed solutions. Unlike the conventional metric of flux decline, which measures the response of a process to fouling, the proposed method quantifies the foulant accumulation. Foulant accumulation is parameterized by two variables, cake structural parameter and hydraulic diameter, that are calculated from flux measurements using a model for salt and water transport through fouled reverse osmosis (RO) and forward osmosis (FO) membranes, including dispersive mass transfer in the FO membrane support layer. Model results show that pressure declines through the foulant layer and can, in FO, reach negative absolute values at the membrane. Experimental alginate gel fouling rates are measured within a range of feed ionic compositions where cake hydraulic resistance is negligible. Using both flux decline and cake structural parameter as metrics, the effect of feed salinity on RO fouling is tested and RO is compared to FO. When RO is fouled with alginate, feed salinity and membrane permeability affect flux decline but not foulant accumulation rate. Between $\mathrm{FO}$ and $\mathrm{RO}$, the initial rates of foulant accumulation are similar; however, FO exhibits slower flux decline, which causes greater foulant accumulation over time. The new methodology enables meaningful quantification and comparison of fouling rates with the aim of improving fundamental understanding of fouling processes.
\end{abstract}

Keywords: alginate, desalination, fouling, forward osmosis, reverse osmosis

\section{Nomenclature}

A Active layer permeability $[\mathrm{m} / \mathrm{s}-\mathrm{Pa}]$

$B \quad$ Salt permeation coefficient of active layer $[\mathrm{m} / \mathrm{s}]$

*Address all correspondence to lienhard@mit.edu 
$C$ Concentration $\left[\mathrm{mol} / \mathrm{m}^{3}\right]$

$D \quad \mathrm{NaCl}$ diffusion coefficient $\left[\mathrm{m}^{2} / \mathrm{s}\right]$

$D_{h} \quad$ Hydraulic diameter [m]

$J_{s} \quad$ Salt molar flux $\left[\mathrm{mol} / \mathrm{m}^{2}-\mathrm{s}\right]$

$J_{w} \quad$ Water flux $[\mathrm{m} / \mathrm{s}]$

$K \quad$ Salt-in-membrane sorption coefficient [-]

L $\quad$ Effective capillary length $[\mathrm{m}]$

$P \quad$ Gauge pressure $[\mathrm{Pa}]$

$R \quad$ Salt rejection [-]

$S \quad$ Structural parameter $[\mathrm{m}]$

T Absolute temperature $[\mathrm{K}]$

$V \quad$ Volume $\left[\mathrm{m}^{3}\right]$

$d_{p} \quad$ Particle diameter $[\mathrm{m}]$

$f_{d} \quad$ Darcy friction factor [-]

$k \quad$ Mass transfer coefficient $[\mathrm{m} / \mathrm{s}]$

$l \quad$ Channel length $[\mathrm{m}]$

$n \quad$ Exponent in Eq. A.2 [-]

$s \quad$ Salt mass fraction [-]

$v \quad$ Velocity $[\mathrm{m} / \mathrm{s}]$

$x \quad$ Distance coordinate perpendicular to membrane $[\mathrm{m}]$

Re Reynolds number [-]

Sc Schmidt number [-]

Sh Sherwood number [-]

Greek

$\alpha \quad$ Dispersivity, as defined in Eq. 14 [m] 
$\delta \quad$ Thickness [m]

$\epsilon \quad$ Porosity [-]

$\gamma \quad$ Surface tension $[\mathrm{N} / \mathrm{m}]$

$\mu \quad$ Dynamic viscosity [Pa-s]

$\pi \quad$ Osmotic pressure $[\mathrm{Pa}]$

$\rho \quad$ Density $\left[\mathrm{kg} / \mathrm{m}^{3}\right]$

$\sigma \quad$ Inhomogeneity factor [-]

$\tau \quad$ Tortuosity [-]

Subscripts

$0 \quad$ Foulant-free

A Active layer

C Cake layer

$D \quad$ Draw boundary layer

F $\quad$ Feed boundary layer

$S \quad$ Support layer

a Apparent

$b \quad$ Interface between support layer and draw

c Interface between cake and feed

d Draw

f $\quad$ Feed

$m \quad$ Interface between membrane and cake

$p \quad$ Permeate

$s \quad$ Interface between active layer and support layer

$w \quad$ Water

eff Effective 


\section{Introduction}

Although osmotic separation processes such as reverse osmosis (RO) are the most energyefficient water treatment technologies for a wide range of water compositions $[1,2,3]$, they are plagued by membrane fouling. A recent review by She et al. [4] discussed a range of fouling types, flux decline models, and mitigation methods. To understand the factors that govern membrane fouling and develop mitigation strategies, many studies have compared different processes, coatings, etc. using flux decline as a metric. Flux decline (the change in flux due to fouling divided by the initial flux) quantifies the effect of fouling on the productivity of a given process, but does not give any insight into the accumulation of foulant itself. Therefore, when studies compare diverse processes such as RO and forward osmosis (FO) using flux decline alone, differences in the response of the processes to foulant accumulation preclude meaningful comparisons. In order to translate experimental results into fundamental understanding of membrane fouling, a deposit-centric, in situ fouling quantification method is needed.

In this paper, a method is outlined for quantifying porous foulant accumulation on semipermeable membranes in terms of two parameters that capture both osmotic and hydraulic causes of flux decline. FO and RO models are developed, although other osmotic processes (assisted forward osmosis, etc.) could be treated similarly if the active layer is facing the feed. The type of fouling considered is porous fouling, which could consist of biofouling, organic fouling, or inorganic fouling so long as no crystals pierce the membrane's active layer and the active area is not blocked by oil deposition or crystal growth. Here, alginate is used as a model porous foulant because of its gelation in the presence of calcium ions and the strong dependence of its material properties on its ionic environment. The proposed method is used to quantify fouling in experimental RO and FO. 


\subsection{Limitations of flux decline}

Many fouling studies are concerned with comparisons, such as the relative fouling propensity of FO vs. RO [5], pressurized vs. unpressurized FO [6, 7], or new membrane coatings vs. commercial membranes [8]. Although flux decline comparisons often keep initial flux constant because of the dependence of fouling rate on flux [9], differences in membrane properties and solution composition can lead to differences in flux decline between experiments even if the foulant layers are identical in size and structure. Flux decline effectively measures the response of the system to fouling; however, it can only hint at the quantity of accumulated foulant.

As an example, Lee et al. [5] compare fouling with colloidal silica and various dissolved organics in $\mathrm{FO}$ and $\mathrm{RO}$, and find that $\mathrm{FO}$ has more severe flux decline in most cases. However, they claim that the faster flux decline in FO is primarily due to the increase in osmotic pressure near the membrane that is enhanced by reverse salt diffusion from the draw solution to the feed solution. Because of the reverse salt diffusion in FO, no conclusion could be drawn about the relative accumulation rates of foulants in $\mathrm{FO}$ and $\mathrm{RO}$ by observing flux decline alone. Rather than continue to make comparisons in terms of flux decline and speculate on how those results relate to fouling propensity, this paper provides a framework using flux measurements to quantify the amount of foulant accumulated as a function of time.

\subsection{Existing methods of quantifying fouling}

Some methods have been developed based on the hydraulic resistance to flow through the porous foulant cake. Hydraulic resistance is generally the main cause of flux decline for membranes with large pores (e.g., ultrafiltration), which do not reject small dissolved species, but this assumption is sometimes extended to salt-rejecting membranes such as RO. For example, Farias et al. [10] quantify fouling with a "membrane fouling index" based on 
a (hydraulic) resistance in series model presented by Nguyen et al. [11] for ultrafiltration membranes. However, methods such as this neglect to account for another important factor in flux decline of semipermeable membranes: the concentration of dissolved constituents within the cake layer and the resulting increase in osmotic pressure at the membrane.

For salt-rejecting membranes such as $\mathrm{FO}$ and $\mathrm{RO}$, this concentrative mechanism of flux decline has been described by Hoek and Elimelech [12]: When a porous cake layer forms on a salt-rejecting membrane, diffusion of salt away from the membrane must counter the flux of salts toward the membrane due to convection with the feed. In this way, the cake layer causes an increase in the osmotic pressure at the membrane active layer, reducing the driving force for water flux according to the solution-diffusion model [13]. As with more porous membranes, there is also some hydraulic resistance to permeation of water through the foulant cake layer, which is accounted for in the Hoek and Elimelech model [12]. This model quantifies foulant accumulation in terms of the osmotic pressure differential across the cake, which they term "cake enhanced osmotic pressure" (CEOP). The CEOP model is useful when making comparisons at a fixed salinity within a fixed process (e.g., seawater RO). However, changes in salinity will affect the osmotic pressure differential for a given foulant cake, so it cannot be used to compare fouling at different salinities (between wastewater RO and seawater RO, for example).

The path toward a universal model has been laid out by these existing studies, which we build on using a layered transport model that accounts for both hydraulic and concentrative causes of flux decline in FO and RO. We take an approach similar to Nagy's model for flux in unfouled FO membranes [14], but incorporate the presence of a porous foulant layer with gradients in both hydraulic and osmotic pressure. We reduce the problem of quantification to two parameters: cake structural parameter (analogous to the support layer structural parameter in FO membranes) and pore hydraulic diameter. Using this model, fouling tests spanning a range of membranes, processes, and feed compositions can be compared directly 
to improve fundamental understanding of fouling processes. We then experimentally compare accumulation rates of alginate gel fouling across two dimensions that take advantage of the comparison capabilities of the new quantification method: (1) the effect of feed salinity in $\mathrm{RO}$ and (2) the difference between FO and RO with the same feed solution.

\section{Layered transport model for fouled RO and FO}

Modeling the effects of fouling in osmotic separation processes is challenging due to widely-ranging length scales, complex geometries, and spatially-varying material properties. As a simplification, we model quasi-steady, one-dimensional transport of salt and water perpendicular to the membrane in both $\mathrm{FO}$ and $\mathrm{RO}$. Real membranes and foulant layers may have indistinct and/or rough interfaces, but this model divides the flow path into distinct, planar layers, each with its own transport model. In many ways, the model developed here parallels the model of Nagy [14] for FO without fouling. Like Nagy, we model transport of a single salt in one dimension through several layers with their own appropriate models, but we add a layer of porous foulant on the feed-facing side of the membrane and also account for dispersion in the FO membrane support layer.

Figure 1 depicts the layers considered and their respective transport models. Feed and draw/permeate concentrations in the bulk flow are treated as boundary conditions. Because salt and water flows are assumed to be one-dimensional and quasi-steady, the salt flux and water flux can be treated as uniform along the direction of flow.

There are several limitations of the model developed herein. Pore blocking (described in [4]) and active layer damage are not modelled. The PRO orientation (active layer facing the draw) and its unique fouling mechanisms (e.g., suction-induced membrane dryout [15]) are not considered. The model can only be applied to foulants of known pore size or those with relatively large pores (over approximately $20 \mathrm{~nm}$ in diameter). Finally, predicting the deposition rate of foulant material is outside the scope of this paper; rather, the model 
(a) RO

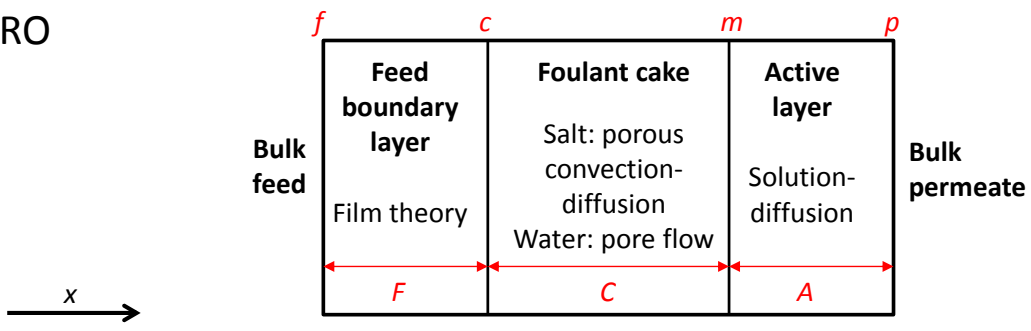

(b) FO

\begin{tabular}{|c|c|c|c|c|c|}
\hline \multirow{3}{*}{$\begin{array}{l}\text { Bulk } \\
\text { feed }\end{array}$} & & & & \multicolumn{2}{|c|}{$b$} \\
\hline & $\begin{array}{c}\begin{array}{c}\text { Feed } \\
\text { boundary } \\
\text { layer }\end{array} \\
\text { Film theory }\end{array}$ & $\begin{array}{l}\text { Foulant cake } \\
\text { Salt: porous } \\
\text { convection- } \\
\text { diffusion } \\
\text { Water: pore flow }\end{array}$ & $\begin{array}{l}\begin{array}{c}\text { Active } \\
\text { layer }\end{array} \\
\text { Solution- } \\
\text { diffusion }\end{array}$ & $\begin{array}{l}\text { Support layer } \\
\text { Porous } \\
\text { convection, } \\
\text { diffusion and } \\
\text { dispersion }\end{array}$ & $\begin{array}{c}\begin{array}{c}\text { Draw } \\
\text { boundary } \\
\text { layer }\end{array} \\
\text { Film theory }\end{array}$ \\
\hline & $F$ & C & $A$ & $S$ & $D$ \\
\hline
\end{tabular}

Figure 1: Summary of layers and their respective models for (a) RO and (b) FO, with water flow from left to right. Boundary conditions (bulk feed, draw and permeate), the positive $x$ direction for all modeling, and subscripts (in red) used to denote layers and interfaces are labeled.

can be used to quantify foulant deposition based on experimentally measured water flux for foulant layers with sufficiently large pores.

In this section, we first address salt and water transport modeling in the foulant cake layer, which is relevant to both FO and RO. We then describe transport through the remaining layers in FO, utilizing the approach of Nagy [14], and develop a similar model for fouled RO. Finally, we suggest a method for solving these systems of equations.

\subsection{Cake structural parameter}

Concentration polarization within the foulant cake layer is a major cause of flux decline for salt-rejecting membranes [12]. The tight pore structure of foulant cakes largely limits flow to the direction perpendicular to the membrane because that path through the cake layer is shortest. As long as the pores in the foulant layer are large enough to permit the passage of dissolved salts, salt is swept into the foulant layer by convection with the water being transported to the membrane. In one-dimensional, quasi-steady operation, the salt 
flux must be equal everywhere along the axis perpendicular to the membrane; however, RO and FO membranes generally exhibit high salt rejection, so the net salt flux is much less than the convective flux toward the membrane. To counter convection, salt diffuses away from the membrane down a concentration gradient. This concentration gradient is the cause of CEOP [12], which in turn causes flux decline due to the increased osmotic pressure at the membrane.

Both convection and diffusion occur through the porous foulant material, which hinders transport by both processes. Neglecting dispersion due to convection through the porous foulant (an assumption justified in Sec. 2.3), the pores can be treated as having an effective length that is greater than the cake thickness, $\delta_{C}$, by a factor of the cake tortuosity, $\tau_{C}$, and an area for diffusion that is reduced by a factor of the foulant porosity, $\epsilon_{C}$. The diffusive flux is thus modified by a factor of $\epsilon_{C} / \tau_{C}$ from the unhindered case, and the effective diffusion distance can be described as $L=\delta_{C} \tau_{C} / \epsilon_{C}$. Gels like alginate can be treated as porous materials because they have a continuous solid phase, with the rest of the volume filled by liquid.

In the support layer of an FO membrane, a similar effective length for diffusion that controls internal concentration polarization (ICP) is called the structural parameter. Therefore, we define the effective thickness of the foulant cake layer as the cake structural parameter, $S_{C}:$

$$
S_{C} \equiv \frac{\delta_{C} \tau_{C}}{\epsilon_{C}}
$$

Having defined a cake structural parameter, salt transport by convection and diffusion through the foulant layer can be modelled the same way as it is in the support layer in FO by Nagy [14]. The cake layer salt transport equation is given in Sec. 2.3. 


\subsection{Cake hydraulic resistance}

Hydraulic resistance to water flow through the cake also contributes to flux decline by reducing the pressure at the feed side of the membrane. Permeation of water through the cake occurs across a pressure drop ${ }^{1}$ that can be estimated using a correlation appropriate for the structure of the porous material. Hoek and Elimelech [12] give a correlation appropriate for an agglomeration of spherical particles; however, we use a capillary model that is more appropriate for a highly hydrated gel such as alginate [16], which is used as a model foulant in our experiments.

In the capillary model, the porous foulant is treated as a bundle of tubes whose pressure drop is prescribed by the Hagen-Poiseuille equation, Eq. 2:

$$
\Delta P=f_{d} \frac{L}{D_{h, C}} \frac{\rho v_{C}^{2}}{2},
$$

where $\Delta P$ is the pressure drop through the bundle, $f_{d}$ is the Darcy friction factor, $L$ is the effective length, $D_{h, C}$ is the hydraulic diameter of pores in the cake, $\rho$ is the fluid density, and $v_{C}$ is the average velocity through the bundle. For pores with diameters on the order of nanometers (true of alginate gels [17], and likely true of most foulant cakes because particles over $0.45 \mu \mathrm{m}$ in diameter generally do not accumulate on the membrane [18], and pores are unlikely to be larger than the particle size), flow through the porous media is laminar. The friction factor can be estimated by the correlation for laminar flow through round tubes,

$$
f_{d}=\frac{64}{\operatorname{Re}_{D_{h, C}}}=\frac{64 \mu}{\rho v_{C} D_{h, C}}
$$

where $\operatorname{Re}_{D_{h, C}}$ is the Reynolds number. Because cakes are not really bundles of round tubes, Eq. 3 is a rough approximation for friction factor. Additionally, we expect a range of pore

\footnotetext{
${ }^{1}$ For salt-rejecting membranes, water flow through the cake is also driven by diffusion, but for the low salt mole fractions typical of desalination feeds, the ratio of diffusive to convective water transport is negligible.
} 
sizes within any foulant cake. However, if $D_{h, C}$ is treated as an effective hydraulic diameter and fit from experimental data using the capillary model (as we do in Appendix A.1), this expression can be used.

The velocity of flow through the pores, $v_{C}$, is inversely related to the porosity, $\epsilon_{C}$, as $v_{C}=J_{w} / \epsilon_{C}$ because of the reduced flow area. The length of flow, $L$, is greater than the foulant thickness, $\delta_{C}$, by a factor of the tortuosity, $\tau_{C}: L=\tau_{C} \delta_{C}$. By substituting these and Eqs. 1 and 3 into Eq. 2, the pressure drop between the feed and the membrane can be related to the structural parameter, pore hydraulic diameter, and water flux:

$$
P_{f}-P_{m}=\frac{32 \mu S_{C} J_{w}}{D_{h, C}^{2}}
$$

\subsection{FO model}

In this section, we present transport equations for salt and water through the remaining layers of the fouled FO membrane. The type of FO modelled in this study transports water from a feed solution into a more concentrated draw solution, which would then typically be regenerated by another separation process [3]. The distance coordinate $x$ is defined as zero at the feed-facing edge of the membrane active layer and positive in the direction of water flow in typical FO and RO operation, i.e., from feed to draw or feed to permeate (direction shown in Fig. 1). Both salt and water fluxes are defined as positive in the positive $x$ direction; as a result, salt flux in FO tends to be negative.

Using stagnant film theory, Nagy [14] models salt transport through both feed and draw boundary layers in FO with the 1-D equation for mass transport by convection and diffusion:

$$
J_{s}=J_{w} C-D \frac{\partial C}{\partial x}
$$

$J_{s}$ is the salt (molar) flux in the $x$ direction in units of $\left[\mathrm{mol} / \mathrm{m}^{2}-\mathrm{s}\right], C$ is the salt concentration in $\left[\mathrm{mol} / \mathrm{m}^{3}\right]$, and $D$ is the diffusion coefficient of salt in water. The concentration differences 
that drive salt diffusion through the boundary layers are often called external concentration polarization, or ECP.

Incorporating a mass transfer coefficient and assuming quasi-steady flow and conserving species (i.e., prescribing constant, spatially uniform $J_{s}$ and $J_{w}$ ), Nagy [14] finds the following equations for salt transport in the feed and draw mass transfer boundary layers in FO:

$$
J_{s}=-J_{w} \frac{C_{c}-C_{f} \exp \left(J_{w} / k_{F}\right)}{\exp \left(J_{w} / k_{F}\right)-1}
$$

where $C_{c}$ is the salt concentration at the outer edge of the foulant cake where it contacts the feed boundary layer. $C_{f}$ is the concentration of the feed solution and $k_{F}$ is the mass transfer coefficient of the feed flow, and:

$$
J_{s}=-J_{w} \frac{C_{d}-C_{b} \exp \left(J_{w} / k_{D}\right)}{\exp \left(J_{w} / k_{D}\right)-1}
$$

where $k_{D}$ is the draw flow mass transfer coefficient, $C_{d}$ is the bulk draw concentration, and $C_{b}$ is the concentration at the draw side of the membrane support layer.

In the active layers of FO or RO membranes, the solution-diffusion model (see Wijmans and Baker [13]) describes the flow of both water and salt. In essence, the solution-diffusion model dictates that both species dissolve into the membrane and diffuse down their chemical potential gradient through a resistance that is characteristic of the membrane active layer. FO water flux depends on both the hydraulic and osmotic pressures according to Eq. 8 [13], which assumes an atmospheric pressure draw:

$$
J_{w}=\left.A\right|_{C_{m}}\left[P_{m}-\left(\left.\pi\right|_{C_{m}}-\left.\pi\right|_{C_{s}}\right)\right]
$$

where $A$ is the water permeability of the membrane active layer (see Appendix B.1 for a discussion of the concentration-dependence of $A$ ), $C$ is the salt concentration, $P$ and $\pi$ are 
hydraulic (gauge) and osmotic pressures, and subscripts $m$ and $s$ refer to the feed-facing and draw-facing sides of the active layer, respectively. Typically, the pressure in FO is close to atmospheric on both sides, but fouling may reduce the pressure at the membrane relative to that of the bulk feed. The pressure drop for convection of water through the porous structure of the support layer is assumed to be negligible, so the pressure behind the active layer is equated in Eq. 8 to the (assumed atmospheric) pressure of the bulk draw.

Salt flux (positive defined as into the draw) is given by the solution-diffusion model [13] as:

$$
J_{s}=-B\left(C_{s}-C_{m}\right)
$$

where $\mathrm{B}$ is the salt permeation coefficient of the active layer. To be precise, Eqs. 8 and 9 are a simplified form of the solution-diffusion model that applies when the driving force across the active layer is smaller than about 100 bar, as it would be in a typical desalination system, due to the linearization mentioned above [13]. Even when a large osmotic pressure gradient is applied to an FO membrane, the osmotic pressure difference across the active layer tends to be dwarfed by the one across the support layer (see Sec. 4.1).

Nagy [14] gives the following equation for salt flux due to convection and diffusion through the porous support layer:

$$
J_{s}=-J_{w} \frac{C_{b}-C_{s} \exp \left(J_{w} S_{S} / D_{S}\right)}{\exp \left(J_{w} S_{S} / D_{S}\right)-1}
$$

where $S_{S}$ is the support layer sturctural parameter,

$$
S_{S} \equiv \frac{\delta_{S} \tau_{S}}{\epsilon_{S}}
$$

However, based on our measurements of unfouled FO membrane flux (see Appendix A.2.1 and Fig. A.10), it would appear that, at higher water fluxes, dispersion is an important 
mechanism of salt transport in the support layer of the FO membrane that has been neglected in prior modeling. Dispersion occurs in convection-diffusion processes through relatively large pores at relatively high flow rates [19], such as in groundwater flows. If dispersion is included, the support layer salt transport equation becomes Eq. 12:

$$
J_{s}=-J_{w} \frac{C_{b}-C_{s} \exp \left(J_{w} S_{S} / D_{S, \text { eff }}\right)}{\exp \left(J_{w} S_{S} / D_{S, \text { eff }}\right)-1}
$$

where $D_{S \text {,eff }}$ is the effective diffusion coefficient, which encompasses the effect of dispersion.

When dispersion is accounted for, the effective diffusion coefficient increases with increasing flux. Perkins and Johnston [19] give the following equation for the apparent diffusion coefficient $D_{a}$ (which we will relate to $D_{S \text {,eff }}$ ) for longitudinal dispersion in random packs of spheres:

$$
D_{a}=\left.D_{a}\right|_{v=0}+0.5 \sigma d_{p} v
$$

where $\left.D_{a}\right|_{v=0}$ is the apparent diffusion coefficient in the porous medium in the absence of convection-driven dispersion, $\sigma$ is the inhomogeneity factor of the porous medium $(\sigma=3.5$ would be typical for a random pack of spheres [19]), $d_{p}$ is the diameter of particles in the pack, and $v$ is an average flow velocity inside the pores. Although the support layer is not really a random pack of spheres, the dispersivity can be fit from experimental data, as we do in Appendix A.2.1.

To translate the apparent diffusivity (Eq. 13) into the effective diffusion coefficient in Eq. 12, we first note that the effective diffusion coefficient in the support layer is $D_{S \text {,eff }}=$ $\left(\tau_{S} / \epsilon_{S}\right) D_{a}$ and that the effective diffusion coefficient without dispersion is $\left.D_{S \text {,eff }}\right|_{J_{w}=0} \equiv$ $D=\left.\left(\tau_{S} / \epsilon_{S}\right) D_{a}\right|_{v=0}$. If we approximate the flow speed inside the porous support layer as $v \approx J_{w} \tau_{S} / \epsilon_{S}$, the resulting effective diffusion coefficient for the support layer can be expressed as:

$$
D_{S, \text { eff }}=D_{S}+\alpha J_{w},
$$


where

$$
\alpha \approx 0.5 \sigma d_{p}\left(\frac{\tau_{S}}{\epsilon_{S}}\right)^{2}
$$

and $d_{p}$ is the equivalent particle diameter of the porous support layer. Short of numerically simulating dispersion based on measured support layer microstructure, accurate calculation of $\alpha$ may be all but impossible. However, dispersivity can be fit from flux measured in foulant-free FO experiments and Eq. 15 can be used to make sure the fitted dispersivity is within a reasonable range. As described in Appendix A.2.1, the value of dispersivity fit from our foulant-free FO flux measurements across a wide range of feed and draw concentrations was $\alpha=1.65 \times 10^{-4} \mathrm{~m}$, which corresponds to $\sigma d_{p} \approx 6.5 \mu \mathrm{m}$ for the product of support layer pore diameter and inhomogeneity factor. SEM and optical micrographs of the same CTA FO membrane used in the present experiments show support layer pore diameters on the order of $10 \mu \mathrm{m}[20]$, so we would expect to see some enhancement of diffusion.

Salt transport through the porous cake layer occurs through convection and diffusion, so we utilize the definition of the cake structural parameter (Eq. 1) to create a salt flux equation analogous to Eq. 12, but for the foulant cake layer:

$$
J_{s}=-J_{w} \frac{C_{m}-C_{c} \exp \left(J_{w} S_{C} / D_{C}\right)}{\exp \left(J_{w} S_{C} / D_{C}\right)-1}
$$

Here, $D_{C}$ is the diffusion coefficient at a representative cake layer concentration without dispersion. Dispersion is neglected in modeling salt transport through the cake layer because, as Eq. 15 shows, dispersivity is proportional to pore diameter, and the pore diameter of the alginate gels considered in the experimental portion of this study have pore diameters on the order of $10 \mathrm{~nm}$ (see Fig. A.9). The ratio $\tau_{S} / \epsilon_{S}$ is also small relative to the support layer. At the fluxes considered in this study (and common in membrane desalination processes), the increase in effective diffusion coefficient due to dispersion through the alginate gel layer should be negligible. However, for other foulant cakes with lower porosity and larger pore 
diameter, the possibility of dispersion should be evaluated using Eqs. 14 and 15.

If the concentration of the feed and draw streams are known, nine unknown variables remain: $C_{c}, C_{m}, C_{s}, C_{b}, J_{w}, J_{s}, P_{m}$, and the foulant parameters $D_{h, C}$ and $S_{C}$. So far we have presented just seven equations: Eqs. 6, 7, 9, 12, and 16 for salt transport and Eqs. 4 and 8 for water. When the pore size of the foulant cake, $D_{h, C}$ is known, the water and salt fluxes can be predicted for a given cake stuctural parameter, $S_{C}$, or $S_{C}$ can be calculated based on measured water flux. A protocol for estimating the foulant pore diameter is given in Appendix A.1, and results are provided for alginate. Alternatively, if the pore size is large enough, the hydraulic pressure drop through the foulant cake can be neglected, and the equation $P_{m}=P_{f}$ added.

A method for solving this system of equations and the necessary correlations for mass transfer coefficients and osmotic pressure are laid out in Sec. 2.5.

\subsection{RO model}

The model for transport through the layers of a fouled RO membrane closely parallels the FO model.

The equation for salt transport in the RO feed boundary layer is identical to the one for FO (Eq. 6). However, unlike FO, concentration polarization in and behind the support layer in $\mathrm{RO}$ can be neglected. Although permeate flowing along the permeate channel may have a different concentration than the permeate coming through the membrane in modules with significant recovery, the high salt rejection of commercial RO membranes leads to a very low permeate salinity and thus negligible concentration polarization on the permeate side.

Equations for salt and water flux through the cake layer in $\mathrm{RO}$ are identical to those for FO (Eqs. 4 and 16).

Salt and water flux through the membrane (Eqs. 17 and 18) are again governed by the solution-diffusion model [13], but the boundary conditions on the back side are the permeate 
pressure (assumed to be atmospheric) and concentration:

$$
J_{s}=B\left(C_{m}-C_{p}\right)
$$

and

$$
J_{w}=\left.A\right|_{C_{m}}\left[P_{m}-\left(\left.\pi\right|_{C_{m}}-\left.\pi\right|_{C_{p}}\right)\right]
$$

where the subscript $p$ refers to the permeate stream. As in FO, the water permeability as a function of salt concentration can be calculated from Eq. B.1. Rather than specifying a permeate salinity, as we do for the draw in FO, permeate salinity is explicitly calculated from the salt and water fluxes:

$$
C_{p}=\frac{J_{s}}{J_{w}}
$$

When feed concentration and pressure are known, $C_{c}, C_{m}, C_{p}, J_{w}, J_{s}, P_{m}, D_{h, C}$, and $S_{C}$ make up eight unknowns. With just six equations $(4,6$, and 16-19), it is insufficient to specify just the flux or structural parameter. As with FO, a known relationship between foulant pore diameter and local conditions can add one equation. Alternatively, in $\mathrm{RO}, C_{p}$ can be measured.

\subsection{Solution method}

In the model of Nagy [14] for unfouled FO, the multiple equations for $J_{s}$ are combined into one through a mass transfer resistance in series model, but the complexity of this equation increases with the number of layers considered by the model. The complexity is further increased by the current model's consideration of the hydraulic resistance of the foulant layer and dispersive salt transport (i.e., effective diffusion coefficient dependence on flux) in the support layer. We have also relaxed the assumption of ideal solution behavior to enable the present model to be used with highly saturated FO draw solutions. Some other models combine layers into an algebraic equation by approximating the salt flux as zero everywhere 
[4], but these models do not account for salt back-diffusion in FO or its effect on foulants whose properties depend on solution ionic composition. To solve these systems of equations, we combine the equations for salt flux into one matrix equation and the equations for water flux into one algebraic equation. These two equations must then be solved simultaneously (we use MATLAB's fsolve). The matrix equations for RO and FO with and without fouling are provided in Appendix C for convenience.

To apply the model, mass transfer coefficient correlations are needed for the feed and draw boundary layers. In evaluating the model, we use mass transfer coefficients measured experimentally with the apparatus configured for $\mathrm{RO}$ as detailed in Appendix A.3. As the foulant cake grows large and begins to encroach on the channel, the mass transfer coefficient may change due to the increasing cross-flow velocity (assuming fixed feed flow rate) as well as changes to the flow pattern when the cake contacts the feed spacer. Future work may address these complexities; for now, the cake layer is assumed to be thin compared to the feed channel and the mass transfer coefficients are assumed to be constant.

Solution properties are also needed to evaluate the model. We use Pitzer's model for electrolyte solutions (see, e.g., $[21,22,23])$ to calculate sodium chloride solution osmotic pressure and density as a function of concentration. Due to the low compressibility of water [24], the high pressures utilized in RO systems are not expected to significantly affect the physical properties of the solutions. Diffusion coefficients are taken from data in [25] (see Appendix B.2).

Membrane properties can be fit from experimental data or estimated from published test results. In Appendix A.2, we demonstrate how the salt permeation coefficients were measured experimentally and calculated from the test results supplied with the membranes and how the FO membrane's support layer structural parameter and dispersivity were fit. In this study, the membrane properties are assumed to vary between membrane samples and one property is fit for each sample from the flux measured just after the foulant was added, 
and before any significant cake layer could form. In RO, water permeability is fit for each membrane. In FO, we fit for the dispersivity because of its strong dependence on support layer properties (Eq. 15).

Finally, in order to relate foulant accumulation to flux decline, pore diameter must be evaluated. We show in Appendix A.1 that for a sufficiently high ratio of sodium to calcium ion concentration, the alginate used as a model porous foulant in this study has sufficiently large pores that the hydraulic pressure drop across the cake is small in comparison to the cake enhanced osmotic pressure. Based on pore size measurements at high sodium:calcium concentration ratios (see Appendix A.1), we use an estimated pore diameter of $20 \mathrm{~nm}$ to calculate cake structural parameter from experimental flux measurements.

\section{Experiment}

$\mathrm{RO}$ and FO fouling experiments are performed to validate and test the proposed fouling quantification method. Transmembrane flux is recorded over time to monitor the evolution of the cake structural parameter in RO and FO due to fouling with feed solutions containing sodium alginate, calcium chloride, and sodium chloride. Flux is calculated from the rate of change in permeate or draw mass in $\mathrm{RO}$ and $\mathrm{FO}$, respectively, while temperature, pressure, and cross flow velocity are fixed. Using this experiment and the layered transport model, we compare foulant accumulation as cake structural parameter between FO and RO and across a range of salinities $(2-3.7 \% \mathrm{NaCl})$ in $\mathrm{RO}$. Salt concentrations are chosen to be within the range where hydraulic pressure drop through the foulant cake (Sec. 2.2) is small compared to the CEOP. Initial flux is kept constant between different trials.

\subsection{Apparatus}

The experimental apparatus, which can be configured for $\mathrm{RO}$ or FO, is illustrated in Fig. 2. 


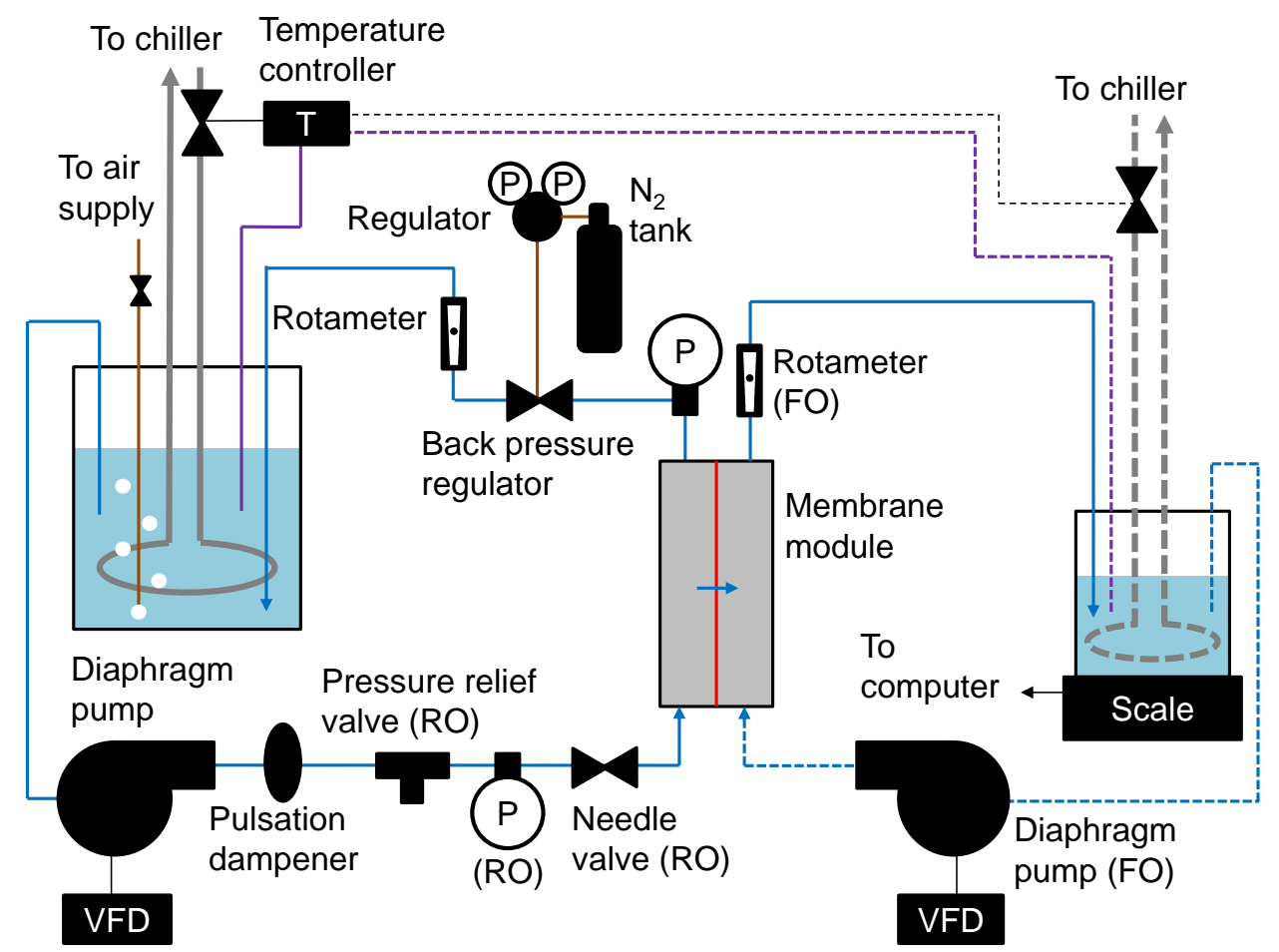

Figure 2: Schematic diagram of the experimental apparatus which can be operated in RO or FO. Some elements (labeled) are only used for RO or FO; dashed lines represent flows only present in FO operation. Purple lines represent thermocouples.

In our custom-built membrane module, the feed channel is cut $1 \mathrm{~mm}$ deep to roughly approximate flow conditions in spiral-wound RO elements, and is equipped with a 0.79 mm-thick non-woven feed spacer (Sterlitech 31 mil diamond). The draw/permeate channel is also $1 \mathrm{~mm}$ deep and uses two $0.43 \mathrm{~mm}$-thick non-woven feed spacers (Sterlitech $17 \mathrm{mil}$ diamond) in FO operation; in $\mathrm{RO}$ operation, it is filled with five layers of permeate spacer mesh cut from a low-pressure RO module. The channels are $8 \mathrm{~cm}$ long and $3 \mathrm{~cm}$ wide, which is comparable to the channel area in previous controlled fouling studies $[5,26]$. Three nested O-rings, which contact each side of the membrane and outside the membrane, rest in grooves designed to minimize membrane damage and provide sealing that ensures very good overall salt rejection (see Appendix A.2) for a membrane module of this size. Details of module design are provided in [15]. 
Flux in $\operatorname{lmh}\left(\mathrm{L} / \mathrm{m}^{2}-\mathrm{hr}\right)$ is calculated from the change in permeate or draw mass during 15 minute intervals. Mass is recorded every second with an Ohaus Scout Pro digital scale of $6 \mathrm{~kg}$ capacity and $0.1 \mathrm{~g}$ repeatability.

Temperature is maintained at $20 \pm 1^{\circ} \mathrm{C}$ by a temperature controller (Omega) and stainless steel cooling coils, which are supplied with chilled water and immersed in the feed and draw tanks. Volume displaced by the draw cooling coil, inlet, and outlet is accounted for in the data analysis. Diaphragm pumps (Hydracell) are driven with variable frequency drives (VFDs) to set the flow rate. A pulsation dampener steadies the flow rate on the feed side; in FO, the need to measure changes in draw volume precludes the use of a pulsation dampener, and the draw flow oscillates around a prescribed mean. Feed pressure is controlled with a back pressure regulator (Equilibar).

Rather than controlling draw and feed concentration, gradual variation is allowed and accounted for in the feed and draw salinities input to the model, as we discuss in Appendix A.4. Conductivity is measured with a Hach conductivity meter, and sodium chloride concentration is interpolated from tabulated data in [27]. The feed is kept well-mixed by a slow stream of air bubbles, which also serve to improve thermal control of the feed by increasing the heat transfer coefficient on the cooling coil [28]. The draw is not actively mixed, but the inlet to the draw pump is placed halfway up the draw tank so that if the tank becomes stratified, draw is taken from the middle of any salinity and temperature gradients.

The maximum system pressure ${ }^{2}$ is 69 barg (1000 psig), allowing for testing of solutions comparable in osmotic pressure to seawater. Aside from diaphragms, all material in contact with high pressure fluid is 316 stainless steel for good corrosion resistance.

\subsection{Membranes}

For RO tests, high-rejection thin-film composite polyamide membranes (Dow FILMTEC SW30HR) are used. For the FO test, an asymmetric cellulose triacetate membrane (Hydra-

\footnotetext{
${ }^{2}$ The pressure unit "barg" refers to bar gauge, or differential pressure with respect to atmospheric pressure.
} 
tion Technology Innovations CTA-ES) was used in FO mode (active layer facing the feed). Property characterization for both membranes is described in Appendix A.2. In contrast to a previous study that compared RO and FO fouling using the same FO membrane [5], the present study uses different membranes for primarily practical reasons: RO membranes are not effective in FO operation because of the high support layer structural parameter, while the chosen FO membranes, rated for just 0.69 bar (10 psi) of transmembrane pressure [29], are not designed to withstand the pressures typical of the RO process. In addition, membrane surface chemistry has a limited effect on foulant cake evolution beyond the adsorbed layer, as shown by Wu et al. [30].

\subsection{Model porous foulant}

Sodium alginate, a polysaccharide composed of mannuronic and guluronic acids, is used as a model porous foulant because polysaccharides are an important component of RO membrane fouling in both wastewater and seawater applications [31] and because it exhibits similarities to the extracellular polymeric substances (EPS) that form a cake layer in biofouling [32]. As described in [33, 34], the polyguluronates in alginic acid complex with calcium and other multivalent ions [35], which are present in most water sources, causing dissolved alginate that concentrates near the membrane to form a cross-linked gel. The sodium alginate used here (Sigma-Aldrich) has a molecular weight rating of 80,000-120,000 $\mathrm{g} / \mathrm{mol}$.

The pore diameter of the alginate gel determines the hydraulic resistance to flow through the foulant cake and is a necessary parameter for computation of the cake layer structural parameter. Many studies have characterized alginate gel pore size, but the range of measurements is wide due to differences in the alginate molecules themselves (which vary in molecular weight, guluronate fraction, and monomer ordering), gelation method, solution ionic composition, and measurement method. For example, Boontheekul et al. [36] characterize an average pore diameter of alginate through thermoporometry, finding a pore diameter of 11.6 
$\pm 0.4 \mathrm{~nm}$ for their high molecular weight $(270,000 \mathrm{~g} / \mathrm{mol})$ alginate gel. Klein et al. [37] who found pore diameters of approximately 6.8-16.6 $\mathrm{nm}$ for three alginates through inverse steric exclusion chromatography. Imaging methods tend to identify larger pore sizes (likely due to the drying necessary), but three diffusion-based methods reviewed in [17] find pore sizes that are also in the $10 \mathrm{~nm}$ range. Shoichet et al. [38] find that a higher degree of cross-linking leads to smaller pores.

Unfortunately (for the aims of this study), permeation hydraulic resistance is related to the square of the hydraulic diameter, and small errors in estimation of the pore radius can lead to large errors in the calculated cake structural parameter when the hydraulic resistance is significant. Future work to characterize pore sizes in alginate formed in RO conditions will be important, but for the present study we will focus on the range in which the hydraulic pressure drop through the cake is very small compared to CEOP (less than about 10\%), so that uncertainties in pore diameter do not significantly influence our results. Appendix A.1 details how this range was bounded.

\subsection{Fouling procedure}

Membranes were soaked in $50 \%$ ethanol, $50 \%$ water solution for five minutes and then in DI water for at least 30 minutes before use. Membranes were installed in the apparatus with the spacers described in Sec. 3.1. Before any foulants were added, RO membranes were compacted and equilibrated with the feed solution for at least two hours or until flux stabilized. FO membranes were equilibrated for at least two hours with the feed solution and saturated $\mathrm{NaCl}$ draw solution (which had been partially degassed to prevent air accumulation in the draw loop). During this period, feed flow rate was maintained at $16.7 \mathrm{~cm} / \mathrm{s}$ to discourage premature fouling. In FO, draw flow rate is maintained at $4.3 \mathrm{~cm} / \mathrm{s}$. In RO, after membrane compaction and equilibration, feed pressure was adjusted to achieve the desired transmembrane flux. 
The fouling procedure was identical between RO and FO experiments aside from the membranes used (see Sec. 3.2) and the driving force for water permeation. To begin fouling, 0.13\% sodium alginate solution (mixed 24 hours prior to ensure complete solvation) was added to the feed tank to reach a final concentration of $200 \mathrm{mg} / \mathrm{L}$. After allowing the alginate to disperse in the air-mixed feed tank for a few minutes, concentrated calcium chloride solution was added to the feed to reach a concentration of $1 \mathrm{mM}$. The feed velocity was then reduced to $8.3 \mathrm{~cm} / \mathrm{s}$, except in the case of the comparison of FO and RO (Sec. 4.3), in which the feed velocity was reduced to $5.6 \mathrm{~cm} / \mathrm{s}$ to accelerate fouling in both tests. Typically, a small, rapid drop in flux occurred just after the alginate and calcium were added and the feed velocity was reduced. We attribute this to increased concentration polarization in the feed channel due to decreased velocity and increased solution viscosity, and the initial flux used to fit permeability and calculate flux decline is chosen to be the flux just after the initial drop. Once fouling has been initiated, transmembrane flux is monitored for ten hours at constant pressure, temperature, and cross-flow velocity.

\section{Results and discussion}

\subsection{Model results: concentration and pressure profiles}

In this section, we calculate concentration and pressure profiles in FO and RO to illustrate the effects of fouling in osmotic processes and help explain the experimental results that follow. Cases considered include high and low concentrations of sodium chloride with $1 \mathrm{mM}$ calcium chloride to show different fouling regimes that depend on ionic composition in both FO and RO. (Appendix D contains piecewise equations for concentration and pressure based on the layered model.)

At high sodium:calcium ratio, cake hydraulic resistance is negligible and the cake layer affects flux through CEOP. Figure 3 shows osmotic and hydraulic pressure profiles throughout the membrane and surrounding layers in FO and $\mathrm{RO}$ with a foulant accumulation of 

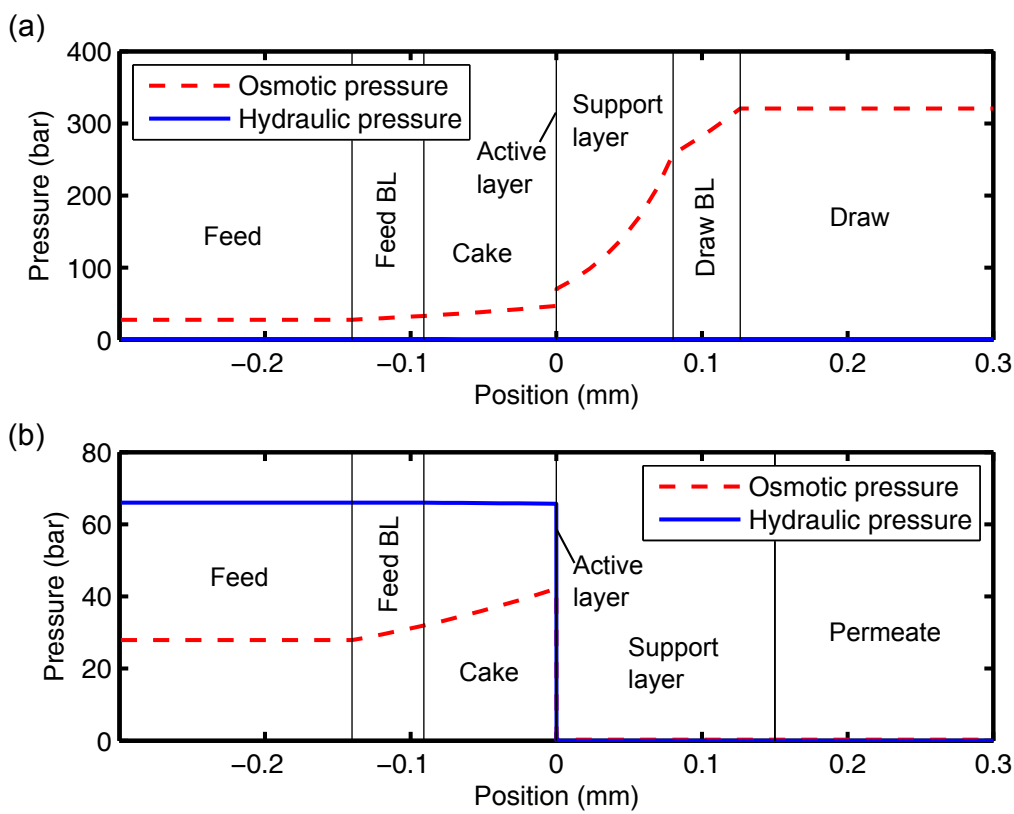

Figure 3: Modelled osmotic and absolute hydraulic pressure profiles for (a) FO and (b) RO with a foulant accumulation of $S_{C}=100 \mu \mathrm{m}$ with $3.5 \%$ wt. $\mathrm{NaCl}$ feed. The label BL stands for "boundary layer."

$S_{C}=100 \mu \mathrm{m}$ (similar to experimental measurements in Secs. 4.2 and 4.3) and a feed solution of $3.5 \%$ wt. $\mathrm{NaCl}$. The modelled FO draw solution was $24 \%$ wt. $\mathrm{NaCl}$ and $\mathrm{RO}$ feed pressure was 66 barg. Pore diameter was assumed to be $20 \mathrm{~nm}$ based on Fig. A.9. For the purpose of illustration only, $\tau_{C} / \epsilon_{C}$ was assumed to be 1.1 to calculate cake thickness from structural parameter.

$\mathrm{RO}$ and FO processes differ greatly in their response to CEOP. In FO, pressure is close to atmospheric everywhere, and flux is driven by the osmotic pressure difference across the membrane active layer. However, as Fig. 3 shows, the driving osmotic pressure difference in FO is dwarfed by the difference across the support layer due to ICP. In contrast, the hydraulic-osmotic pressure difference across the active layer in RO is by far the largest of any layer in the system. When a certain CEOP develops in RO, the driving force for flow across the active layer is reduced by an equal amount (neglecting the small impact of feed ECP). In FO, a small flux reduction due to CEOP causes a significant reduction in the 
(a)

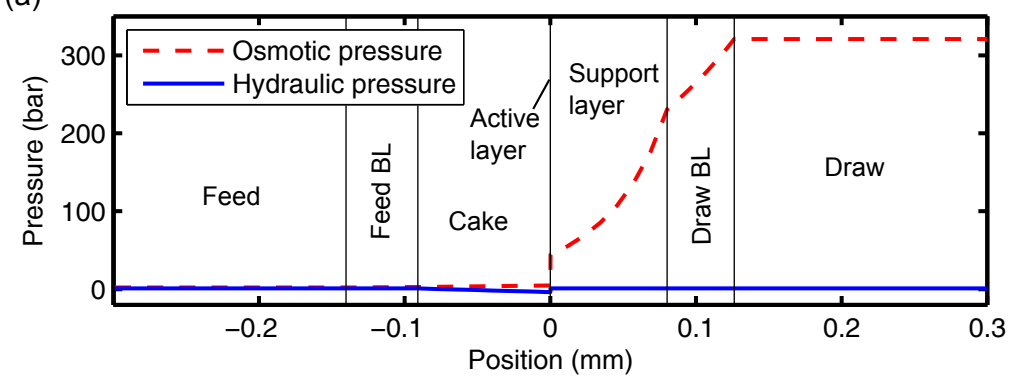

(b)

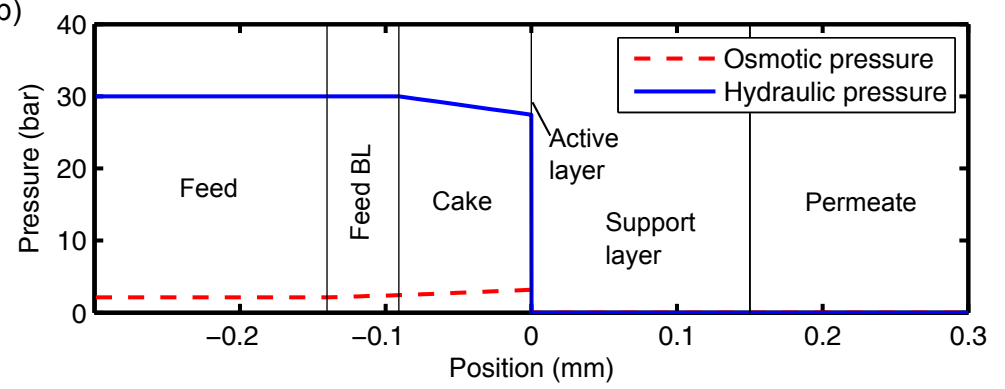

Figure 4: Modelled osmotic and absolute hydraulic pressure profiles for (a) FO and (b) RO with $50 \mathrm{mM}$ $\mathrm{NaCl}$ feed and a foulant accumulation of $S_{C}=100 \mu \mathrm{m}$, showing negative absolute pressure within the cake in the $\mathrm{FO}$ case.

concentration difference across the support layer, so although the osmotic pressure on the feed side of the active layer increases, the draw side osmotic pressure increases almost as much. This phenomenon, referred to as "ICP self-compensation" [4], results in a smaller flux decrease in FO than in RO for the same cake layer. This important difference between these systems' response to fouling is also demonstrated through experimental results in Sec. 4.3.

When the sodium:calcium ratio is lower, the relative importance of hydraulic pressure drop and CEOP are reversed with respect to the high-sodium case. Figure 4 shows concentration and pressure profiles in $\mathrm{FO}$ and $\mathrm{RO}$ for a feed of $50 \mathrm{mM} \mathrm{NaCl}$ and $1 \mathrm{mM} \mathrm{CaCl}$, for which a pore size of $6.6 \mathrm{~nm}$ was estimated from the results in Fig. A.9. FO draw solution was $24 \%$ wt. $\mathrm{NaCl}$ and $\mathrm{RO}$ feed pressure was 30 barg. The feed salinity $(0.27 \%$ wt. $\mathrm{NaCl})$ is so low that concentration polarization in the cake layer causes only a small increase in 
osmotic pressure at the membrane. However, the hydraulic pressure drops by several bars through the cake, reaching a minimum absolute pressure of -3.9 bar at the membrane.

It is possible for negative absolute pressures to develop within the foulant layer because of the small pore size of alginate gels: in a matrix of a highly hydrophilic material like alginate, the interfacial pressure drop of a stable vapor bubble within a pore would scale with $4 \gamma / D_{h, C}$, where $\gamma$ is the surface tension of saline water at the local salinity and $D_{h, C}$ is the pore hydraulic diameter within the cake. For a pore size around $7 \mathrm{~nm}$, the interfacial pressure difference between the inside and outside of a bubble would have to be on the order of 400 bar. In this way, the nano-scale pores of the alginate gel prevent cavitation within the foulant cake even when it is under some tension. Negative pressures have been shown to exist in tree xylem, another liquid-filled nanoporous material subjected to suction [39]. However, alginate fouling has been shown to cause nucleation in the larger, micro-scale pores of the feed-facing support layer of FO membranes in PRO orientation [15]. Large pores or hydrophobic materials in the foulant layer could theoretically act as nucleation sites regardless of membrane orientation, although this has not to our knowledge been demonstrated.

\subsection{Effect of feed salinity in $R O$}

Part of the motivation for developing a universal method for fouling quantification is to be able to compare fouling rates in waters of differing ionic composition. In this section, we examine experimental fouling results for $\mathrm{RO}$ at $\mathrm{NaCl}$ concentrations of $2-3.7 \%$ wt. in terms of both flux decline and cake structural parameter. As in all trials, alginate concentration was $200 \mathrm{mg} / \mathrm{L}$ with $1 \mathrm{mM} \mathrm{CaCl}$. Flux decline due to fouling was calculated from measured flux as $1-J_{w} / J_{w, 0}$, where $J_{w, 0}$ is the foulant-free flux calculated from the layered model with no foulant layer (Eqs. C.3, C.4, C.7, and C.8). The $95 \%$ confidence intervals on the measurements in this section are $\pm 3.6 \%$ for flux decline and $\pm 13 \mu \mathrm{m}$ for cake structural parameter in $\mathrm{RO}( \pm 14 \mu \mathrm{m}$ for the $2 \%$ wt. $\mathrm{NaCl}$ case). Uncertainty analysis is discussed in Appendix E. The RO model was validated against experimental data in Appendix A.1. 
Trials were conducted as close as possible to the same initial flux, but the actual initial flux values were $22.8 \pm 0.4 \mathrm{lmh}$. To minimize the effects of slight deviations in initial flux on the fouling comparison, both flux decline and cake structural parameter are reported as a change from the values at the moment the flux reached $22.4 \mathrm{lmh}$. We have assumed that the period where the fouling accumulation rate is affected by the membrane surface properties is short compared to the time over which the initial flux is calculated (15 minutes). In the present experiments, the foulant layer thickness (on the order of tens or hundreds of micrometers) is much greater than the roughness of the membrane (order $100 \mathrm{~nm}$ in $\mathrm{RO}$ [40] or less in FO [20]) or the pore size of alginate (order $10 \mathrm{~nm}$ ). Additionally, different membrane functional groups that affect initial alginate adsorption rate in RO have been shown not to significantly affect fouling in the long term [30]. Therefore, we focus on the stage of the foulant accumulation process where new foulant is attaching to existing foulant, not the membrane, and thus fouling trials beginning at different fluxes can be compared with respect to an arbitrary "initial" flux.

Figures 5 and 6 show the flux decline and foulant accumulation (as cake structural parameter) of three different feed solutions with $\mathrm{NaCl}$ concentrations of 2.0, 3.1, and 3.7\% wt. at pressures of 36, 64.5, and (again) $64.5 \mathrm{barg}$, respectively. The flux decline results in Fig. 5 follow no clear trend with respect to salinity or pressure because of the variation in permeability (which itself affects flux decline) between membrane coupons. However, Fig. 6 is more illustrative: when flux measurements are used to compute cake structural parameter starting at the same flux, the evolution of the cake structural parameter over time is indistinguishable between the trials (with the exception of a brief excursion around 3 hours of the $3.1 \% \mathrm{NaCl}$ trial, which we attribute to experimental error). By allowing the quantity of foulant to be extracted from flux decline measurements, the layered model shows that within the range of high sodium:calcium ratios considered here, solution ionic composition does not affect alginate gel accumulation rate. Morris et al. [33] find that the bound calcium 


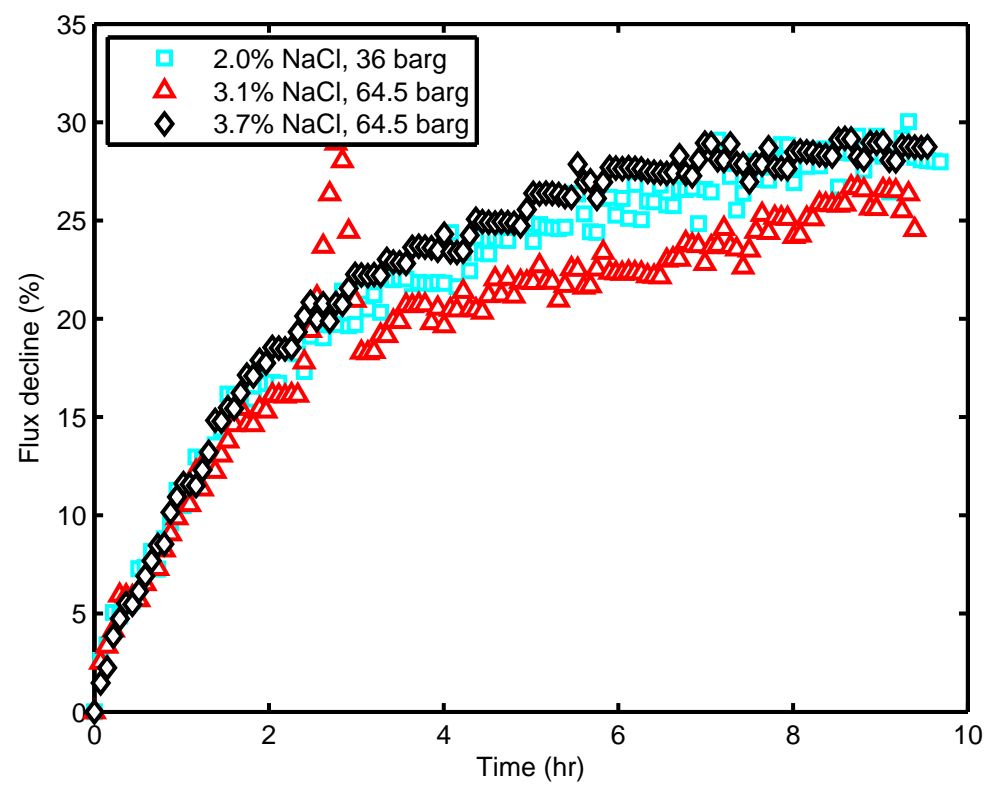

Figure 5: Comparison of flux decline profiles in $\mathrm{RO}$ with feeds of different salinity at different pressures. Permeability varied between coupons and pressures were chosen such that initial flux was $22.8 \pm 0.4 \mathrm{lmh}$. To minimze the effect of initial flux on fouling rate, flux decline is given relative to the moment each run reached a flux of $22.4 \mathrm{lmh}$. Cross-flow velocity was fixed at $8.3 \mathrm{~cm} / \mathrm{s}$. Uncertainty in flux decline is \pm 3.6 percentage points.

fraction asymptotes to a minimum value beyond a certain sodium:calcium ratio, so it is possible that other properties asymptote similarly. However, the bound calcium fraction [33] and intermolecular adhesion forces [26] do vary within a lower range of sodium:calcium ratios, so some change in foulant accumulation rate might be expected with feeds of lower sodium or higher calcium concentration.

Notably, Fig. 6 shows no effect of pressure on foulant accumulation rate, even between pressures of 36 and 64.5 barg. This result contrasts with the findings of Xie et al. [7] on the effect of pressure in forward osmosis, in which confocal laser scanning microscopy was used to show that thicker foulant layers develop in FO at atmospheric pressure than in RO at just 12.5 barg on the same membrane. The apparent effect of pressure on fouling of FO membranes does not seem to extend to RO. 


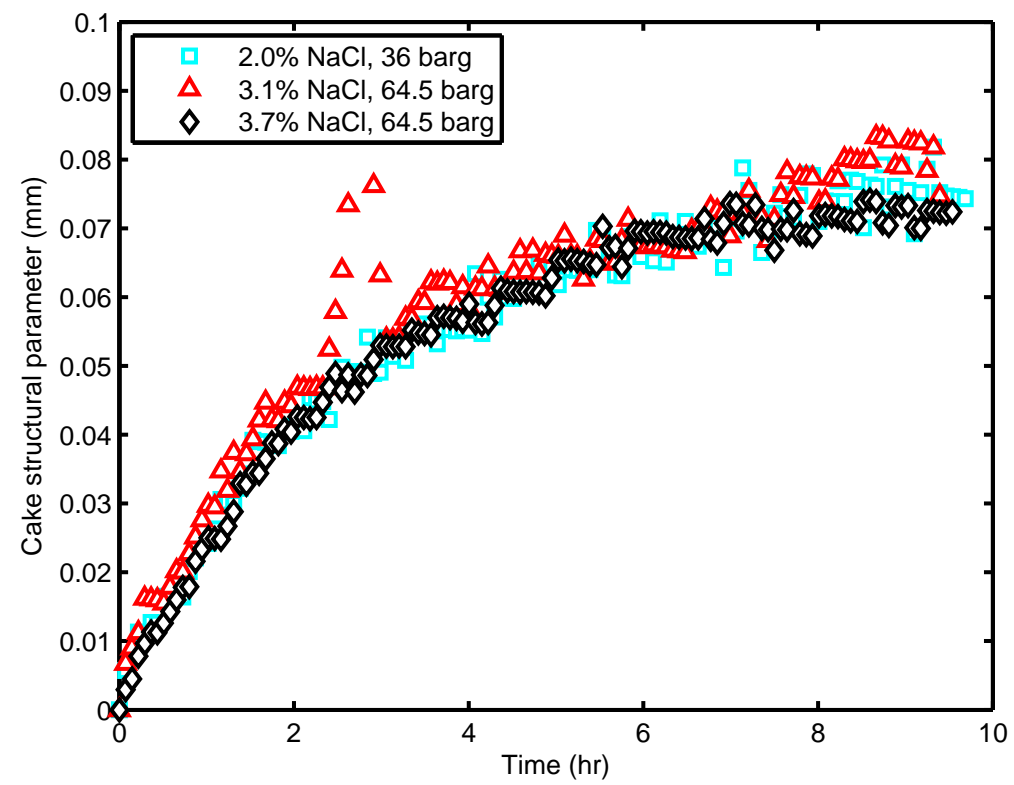

Figure 6: Comparison of calculated foulant accumulation in RO tests with feeds of different salinity on membrane coupons of varying permeability at different pressures, showing strong similarity. The cake structural parameter was calculated using the model presented here for the same tests that exhibited differences in flux decline in Fig. 5. To eliminate the effect of flux on fouling rate, cake structural parameter is given as a change relative to the moment each run reached a flux of $22.4 \mathrm{lmh}$. Uncertainty is $\pm 14 \mu \mathrm{m}$ for $2 \% \mathrm{NaCl}$ is and $\pm 13 \mu \mathrm{m}$ for others.

\subsection{Comparison of $R O$ and $F O$}

Fouling rates in $\mathrm{FO}$ and $\mathrm{RO}$ are compared in this section in terms of flux decline and cake structural parameter to elucidate differences in the response to fouling and the rate of foulant accumulation. For these trials, the $\mathrm{NaCl}$ mass fraction was $3.5 \%$ and the cross-flow velocity was $5.6 \mathrm{~cm} / \mathrm{s}$. Alginate concentration was again $200 \mathrm{mg} / \mathrm{L}$ and $\mathrm{CaCl}_{2}$ concentration was $1 \mathrm{mM}$. RO initial flux was $18.2 \mathrm{lmh}$ (at $50 \mathrm{barg}$ ) and FO initial flux was $18.0 \mathrm{lmh}$, but flux decline and $S_{C}$ are given relative to the moment each run reached a flux of $18.0 \mathrm{lmh}$ to eliminate the effect of initial flux on fouling rate. The $95 \%$ confidence intervals on RO flux decline and cake structural parameter measurements are again $\pm 3.6 \%$ and $\pm 13 \mu \mathrm{m}$, respectively. As discussed in Appendix E, the uncertainty in FO flux decline and cake structural parameter are $\pm 5.7 \%$ and $\pm 40 \mu \mathrm{m}$, respectively. 


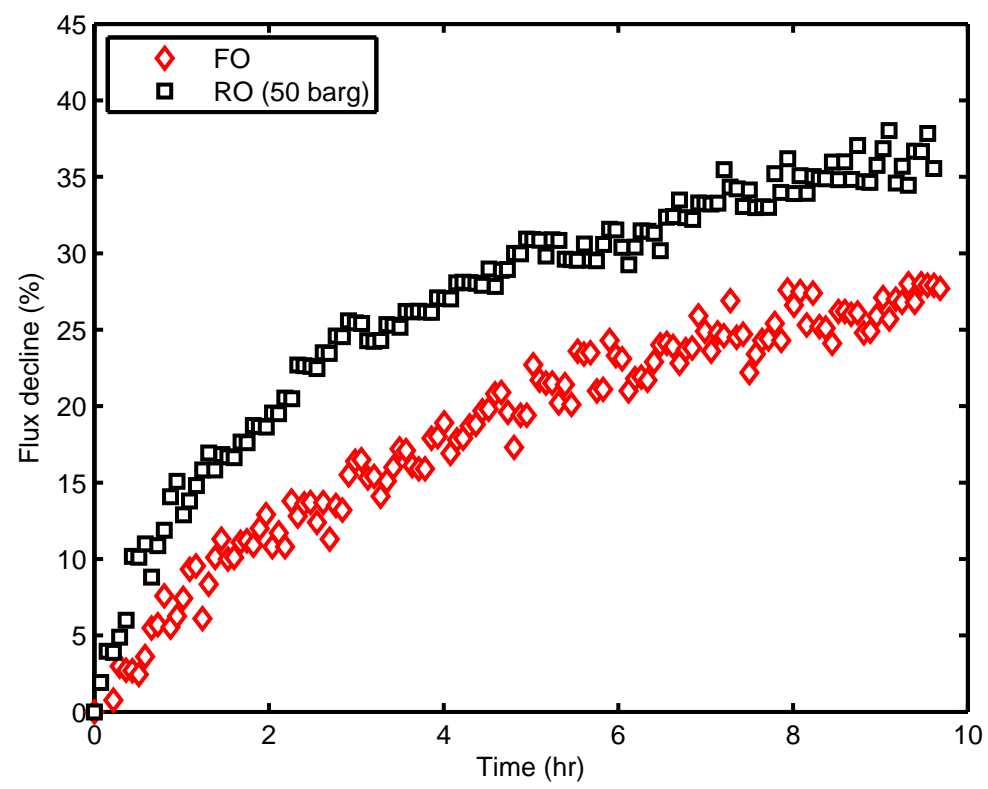

Figure 7: Comparison of flux decline profiles in $\mathrm{RO}$ and $\mathrm{FO}$ with $3.5 \% \mathrm{NaCl}$ feed, showing greater flux decline in RO. RO initial flux was $18.2 \mathrm{lmh}$ and FO initial flux was $18.0 \mathrm{lmh}$, but flux decline is given relative to the moment each run reached a flux of $18.0 \mathrm{lmh}$ to eliminate the effect of initial flux on fouling rate. Feed cross-flow velocity was $8.3 \mathrm{~cm} / \mathrm{s}$ in both trials. $95 \%$ confidence intervals are \pm 3.6 and \pm 5.7 percentage points in $\mathrm{RO}$ and $\mathrm{FO}$, respectively.

As shown in Fig. 7, flux decline is slower in FO than in $\mathrm{RO}$ under the same conditions. However, FO flux decline is expected to be slower because of the ICP self-compensation effect, as discussed in Sec. 4.1. As a result, it is not meaningful to draw a conclusion about the amount of fouling that occurred in FO vs. RO based on flux decline alone.

When fouling accumulation is reported as cake structural parameter (Fig. 8), it is clear that despite its lower flux decline, the FO test resulted in a thicker foulant cake. Initially, the flux and accumulation rate are similar between FO and RO. However, as time progresses, flux drops more rapidly in RO than in FO (Fig. 7), as does the rate of foulant accumulation. This effect can be explained, at least to some extent, through an analogy to heat exchanger fouling made by Qureshi et al. [41], which suggests that RO fouling resistance follows an exponential approach to an asymptotic value at which the rate of deposition (proportional to 


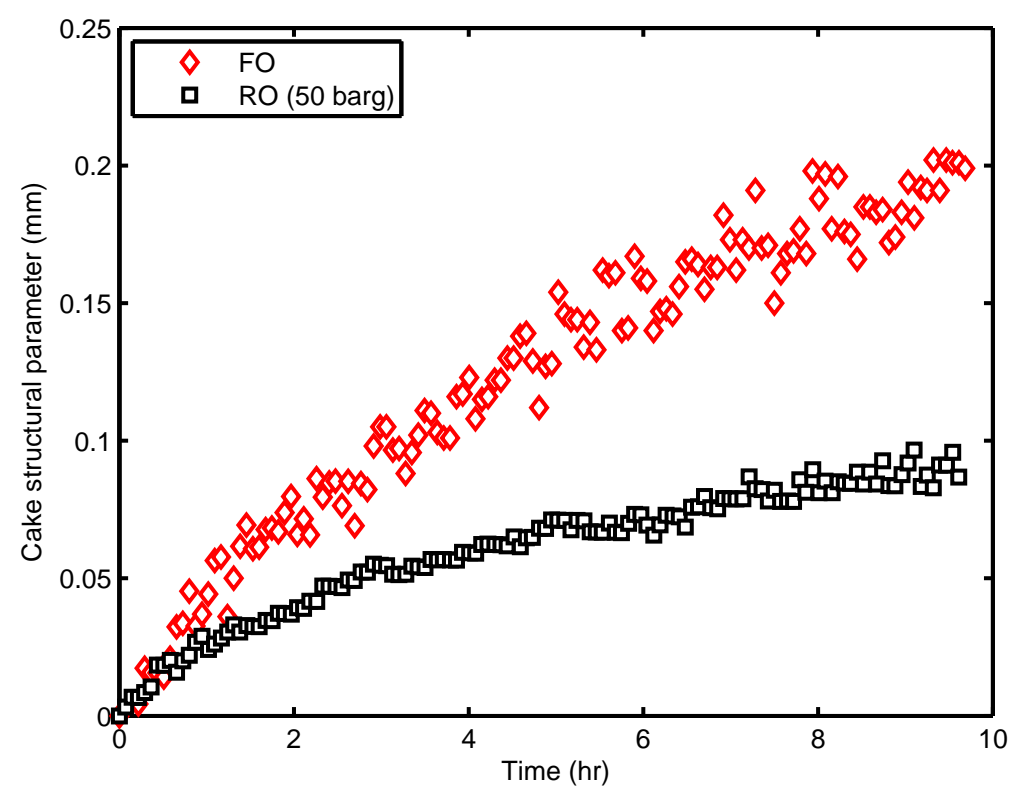

Figure 8: Comparison of calculated foulant accumulation in $\mathrm{RO}$ and $\mathrm{FO}$, showing similar accumulation rates initially but greater accumulation over time in FO. To eliminate the effect of initial flux on initial fouling rate, cake structural parameter is given as a change relative to the moment each run reached a flux of 18.0 lmh. $95 \%$ confidence intervals are $\pm 13 \mu \mathrm{m}$ for RO and $\pm 40 \mu \mathrm{m}$ for FO.

flux) is equal to the rate of removal. According to that model, the total accumulation should be propotional to the time integral of flux minus asymptotic flux, and the process with a slower flux decline (FO, in this comparison) should have a larger cake structural parameter. Xie et al. [7] found a similar result using confocal laser scanning microscopy on alginate gel layers harvested from fouled membranes: when operated under similar conditions with the same initial flux, thicker cake layers developed in FO than in RO; however, that study attributed the thinner layer in RO to compaction by hydraulic pressure. Future work should address whether the disparity in foulant accumulation is solely driven by the difference in flux decline rates of FO and RO systems, or whether other differences contribute as well.

As a means of validating the FO structural parameter model, the foulant cake formed during the FO trial in Fig. 8 was scraped off of the membrane immediately after the trial and weighed at $0.46 \mathrm{~g}$. Assuming the density of the hydrogel is similar to water and the 
tortuosity and porosity are near 1 , the final structural parameter can be estimated from the mass measurement as $190 \mu \mathrm{m}$, which is within $5 \%$ of the final structural parameter calculated from the flux decline (approximately $200 \mu \mathrm{m}$ ).

\section{Conclusion}

In this study, we developed a method of in situ fouling quantification that allows for comparisons of foulant accumulation between diverse processes with different membranes and feed solutions. Foulant accumulation is reduced to two variables (cake structural parameter and foulant pore hydraulic diameter) that can be calculated in RO or FO from experimental flux measurements using a layered model for salt and water transport which includes dispersion in the FO support layer. Notably, the model demonstrates that in FO, under certain conditions, absolute pressure can reach negative values at the membrane. The model was experimentally validated for alginate gel fouling in a range of conditions where cake hydraulic resistance is negligible; future work to characterize the dependence of pore size on ionic environment for various foulants is needed to broaden the applicability of this quantification method.

The proposed method was used to analyze fouling experiments, leading to the following conclusions:

- In waters with a high sodium:calcium concentration ratio, alginate fouling accumulation rates in $\mathrm{RO}$ are independent of salinity and pressure.

- The slower flux decline of FO results in a higher rate foulant accumulation compared to RO.

It is hoped that this quantification method will accelerate progress in understanding fouling, especially as related to claimed differences in fouling propensity between FO and $\mathrm{RO}$, and that it will lead to future capabilities in predictive modeling of fouling. 


\section{Acknowledgements}

We would like to acknowledge support from the King Fahd University of Petroleum and Minerals through the Center for Clean Water and Clean Energy at MIT and KFUPM (Project \#R4-CW-11) and from the MIT Martin Family Society of Fellows for Sustainability. This material is based upon work supported by the National Science Foundation Graduate Research Fellowship Program under Grant No. 1122374. We would also like to thank Gregory Thiel for sharing his implementation of Pitzer's electrolyte solution model for aqueous sodium chloride solution properties and Jaichander Swaminathan for useful discussions.

\section{References}

[1] E. W. Tow, R. K. McGovern, J. H. Lienhard V, Raising forward osmosis brine concentration efficiency through flow rate optimization, Desalination 366 (2015) $71-79$.

[2] G. P. Thiel, E. W. Tow, L. D. Banchik, H. W. Chung, J. H. Lienhard V, Energy consumption in desalinating produced water from shale oil and gas extraction, Desalination 366 (2015) $94-112$.

[3] R. K. McGovern, J. H. Lienhard V, On the potential of forward osmosis to energetically outperform reverse osmosis desalination, Journal of Membrane Science 169 (2014) 245-250.

[4] Q. She, R. Wang, A. G. Fane, C. Y. Tang, Membrane fouling in osmotically driven membrane processes: A review, Journal of Membrane Science 499 (2016) $201-233$.

[5] S. Lee, C. Boo, M. Elimelech, S. Hong, Comparison of fouling behavior in forward osmosis (FO) and reverse osmosis (RO), Journal of Membrane Science 365 (2010) 34 - 39.

[6] Y. Kim, M. Elimelech, H. K. Shon, S. Hong, Combined organic and colloidal fouling in forward osmosis: Fouling reversibility and the role of applied pressure, Journal of Membrane Science 460 (2014) 206 212 .

[7] M. Xie, J. Lee, L. D. Nghiem, M. Elimelech, Role of pressure in organic fouling in forward osmosis and reverse osmosis, Journal of Membrane Science 493 (2015) 748-754.

[8] A. Matin, H. Shafi, M. Wang, Z. Khan, K. Gleason, F. Rahman, Reverse osmosis membranes surfacemodified using an initiated chemical vapor deposition technique show resistance to alginate fouling under cross-flow conditions: Filtration \& subsequent characterization, Desalination 379 (2016) 108 117. 
[9] E. M. Hoek, J. Allred, T. Knoell, B.-H. Jeong, Modeling the effects of fouling on full-scale reverse osmosis processes, Journal of Membrane Science 314 (2008) 33 - 49.

[10] E. L. Farias, K. J. Howe, B. M. Thomson, Spatial and temporal evolution of organic foulant layers on reverse osmosis membranes in wastewater reuse applications, Water Research 58 (2014) 102 - 110.

[11] A. H. Nguyen, J. E. Tobiason, K. J. Howe, Fouling indices for low pressure hollow fiber membrane performance assessment, Water Research 45 (2011) 2627 - 2637.

[12] E. M. V. Hoek, M. Elimelech, Cake-enhanced concentration polarization: A new fouling mechanism for salt-rejecting membranes, Environmental Science and Technology 37 (2003) 5581-5588.

[13] J. Wijmans, R. Baker, The solution-diffusion model: a review, Journal of Membrane Science 107 (1995) $1-21$.

[14] E. Nagy, A general, resistance-in-series, salt- and water flux models for forward osmosis and pressureretarded osmosis for energy generation, Journal of Membrane Science 460 (2014) 71 - 81.

[15] E. W. Tow, M. M. Rencken, J. H. Lienhard V, In situ visualization of organic fouling and cleaning mechanisms in reverse osmosis and forward osmosis, Submitted to Journal of Membrane Science (2016).

[16] A. Karabelas, D. Sioutopoulos, New insights into organic gel fouling of reverse osmosis desalination membranes, Desalination 368 (2015) $114-126$.

[17] C. Simpliciano, L. Clark, B. Asi, N. Chu, M. Mercado, S. Diaz, M. Goedert, M. Mobed-Miremadi, Cross-linked alginate film pore size determination using atomic force microscopy and validation using diffusivity determinations, Journal of Surface Engineered Materials and Advanced Technology 3 (2013) $1-12$.

[18] D. E. Potts, R. C. Ahlert, S. S. Wang, A critical review of fouling of reverse osmosis membranes, Desalination 36 (1981) 235-264.

[19] T. Perkins, O. Johnston, A review of diffusion and dispersion in porous media, Society of Petroleum Engineers Journal 3 (1963) 70-84.

[20] Y. Wang, F. Wicaksana, C. Y. Tang, A. G. Fane, Direct microscopic observation of forward osmosis membrane fouling, Environmental Science \& Technology 44 (2010) 7102-7109.

[21] K. S. Pitzer, Thermodynamics of electrolytes. I. Theoretical basis and general equations, The Journal of Physical Chemistry 77 (1973) 268-277.

[22] G. P. Thiel, J. H. Lienhard V, Treating produced water from hydraulic fracturing: Composition effects on scale formation and desalination system selection, Desalination 346 (2014) $54-69$.

[23] K. H. Mistry, H. A. Hunter, J. H. Lienhard V, Effect of composition and nonideal solution behavior 
on desalination calculations for mixed electrolyte solutions with comparison to seawater, Desalination 318 (2013) $34-47$.

[24] K. G. Nayar, M. H. Sharqawy, L. D. Banchik, J. H. Lienhard V, Thermophysical properties of seawater: pressure dependence, new correlations and a review, Desalination, accepted (2016).

[25] V. Vitagliano, P. A. Lyons, Diffusion coefficients for aqueous solutions of sodium chloride and barium chloride, Journal of the American Chemical Society 78 (1956) 1549-1552.

[26] S. Lee, M. Elimelech, Salt cleaning of organic-fouled reverse osmosis membranes, Water Research 41 (2007) $1134-1142$.

[27] R. Robinson, R. Stokes, Electrolyte Solutions: Second Revised Edition, Dover Publications, Incorporated, 2012.

[28] E. W. Tow, J. H. Lienhard V, Heat transfer to a horizontal cylinder in a shallow bubble column, International Journal of Heat and Mass Transfer 79 (2014) 353-361.

[29] HTI OsMem CTA-ES Membrane Specifications, Hydration Technology Innovations, received in 2014.

[30] J. Wu, A. E. Contreras, Q. Li, Studying the impact of RO membrane surface functional groups on alginate fouling in seawater desalination, Journal of Membrane Science 458 (2014) 120 - 127.

[31] M. T. Khan, M. Busch, V. G. Molina, A.-H. Emwas, C. Aubry, J.-P. Croue, How different is the composition of the fouling layer of wastewater reuse and seawater desalination RO membranes?, Water Research 59 (2014) $271-282$.

[32] Y. Ye, P. L. Clech, V. Chen, A. Fane, B. Jefferson, Fouling mechanisms of alginate solutions as model extracellular polymeric substances, Desalination 175 (2005) 7 - 20.

[33] E. R. Morris, D. A. Rees, D. Thom, J. Boyd, Chiroptical and stoichiometric evidence of a specific, primary dimerisation process in alginate gelation, Carbohydrate Research 66 (1978) $145-154$.

[34] I. Braccini, S. Perez, Molecular basis of Ca2+-induced gelation in alginates and pectins: The egg-box model revisited, Biomacromolecules 2 (2001) 1089-1096.

[35] A. Haug, O. Smidsrod, The effect of divalent metals on the properties of alginate solutions II: Comparison of different metal ions, Acta Chemica Scandinavica 19 (1965) 341-351.

[36] T. Boontheekul, H.-J. Kong, D. J. Mooney, Controlling alginate gel degradation utilizing partial oxidation and bimodal molecular weight distribution, Biomaterials 26 (2005) 2455 - 2465.

[37] J. Klein, J. Stock, K.-D. Vorlop, Pore size and properties of spherical Ca-alginate biocatalysts, European Journal of Applied Microbiology and Biotechnology 18 (1983) 86-91.

[38] M. S. Shoichet, R. H. Li, M. L. White, S. R. Winn, Stability of hydrogels used in cell encapsulation: 
An in vitro comparison of alginate and agarose, Biotechnology and Bioengineering 50 (1996) 374-381.

[39] J. S. Sperry, N. Z. Saliendra, W. T. Pockman, H. Cochard, P. Cruiziat, S. D. Davis, F. W. Ewers, M. T. Tyree, New evidence for large negative xylem pressures and their measurement by the pressure chamber method, Plant, Cell \& Environment 19 (1996) 427-436.

[40] J. S. Louie, I. Pinnau, I. Ciobanu, K. P. Ishida, A. Ng, M. Reinhard, Effects of polyether-polyamide block copolymer coating on performance and fouling of reverse osmosis membranes, Journal of Membrane Science 280 (2006) $762-770$.

[41] B. A. Qureshi, S. M. Zubair, A. K. Sheikh, A. Bhujle, S. Dubowsky, Design and performance evaluation of reverse osmosis desalination systems: An emphasis on fouling modeling, Applied Thermal Engineering 60 (2013) $208-217$.

[42] A. Achilli, T. Y. Cath, A. E. Childress, Power generation with pressure retarded osmosis: An experimental and theoretical investigation, Journal of Membrane Science 343 (2009) 42 - 52.

[43] DOW FILMTEC SW30HR380 Element Product Data Sheet, Dow Water \& Process Solutions, 2015.

[44] C. Koutsou, S. Yiantsios, A. Karabelas, A numerical and experimental study of mass transfer in spacer-filled channels: Effects of spacer geometrical characteristics and Schmidt number, Journal of Membrane Science 326 (2009) $234-251$.

[45] FILMTEC(TM) Reverse Osmosis Membranes Technical Manual, Dow Water \& Process Solutions, 2011.

[46] X. Jin, A. Jawor, S. Kim, E. M. Hoek, Effects of feed water temperature on separation performance and organic fouling of brackish water RO membranes, Desalination 239 (2009) 346 - 359.

\section{Appendix A. Experimental details}

\section{Appendix A.1. Separating hydraulic and concentrative causes of flux decline}

To separate the hydraulic cause of flux decline (drag) from the concentrative cause (CEOP), the feed solution osmotic pressure was varied for a fixed foulant accumulation in a series of RO tests, allowing both cake structural parameter and pore size to be fit from the experimental data. However, due to the complexation of alginate with calcium and the variation in the complexation type with sodium:calcium ratio [33], varying the osmotic pressure by adding sodium and/or calcium salts could change the size of the pores being measured. Instead, the feed osmotic pressure was varied within individual RO tests by 
adding glucose, a small, nonionic solute that should not affect alginate structure.

For these tests, an alginate gel cake was formed over a period of four hours on an RO membrane following the procedure described in Sec. 3.4, except that the feed spacer is removed to expedite fouling. The feed solution was then replaced with a foulant-free feed solution with the same sodium chloride and calcium chloride concentrations as the initial feed. After the flux was again stabilized, the new flux and feed conductivity were recorded. Glucose was added to reach a concentration of first $0.05 \mathrm{M}$, and then $0.1 \mathrm{M}$. After each addition, stable flux and feed conductivity were measured. The flux and feed conductivity for each glucose concentration were run through the FO model, assuming that the dilute glucose in solution behaves as an ideal solute so that glucose and salt concentration profiles could be superposed. Permeation of glucose through the RO membrane was neglected. The cake structural parameter and pore size were calculated from the best fit of flux at the three glucose concentrations $(0,0.05$, and $0.1 \mathrm{M})$ at each sodium:calcium ratio. The slight decrease in flux that occurs with increasing osmotic pressure may have altered the structural parameter and pore size due to the decreasing cake compression, but this effect was neglected here.

To validate the model, the foulant cake was peeled off the membrane at the end of the test with $0.2 \mathrm{M} \mathrm{NaCl}$ and $1 \mathrm{mM} \mathrm{CaCl}_{2}$ and its mass was measured. Assuming the density of the gel is approximately equal to that of water, the thickness was estimated to be $306 \mu \mathrm{m}$. This is $9.3 \%$ greater than the structural parameter fit with the model for this trial of $S_{C}=280$ $\mu \mathrm{m}$, and given that alginate gels are highly hydrated and thus tend to have porosity and tortuosity near unity (see [16]), a measured thickness that is close to the calculated structural parameter provides some validation for the proposed fouling quantification method for RO. Compression of the cake due to flux through it during structural parameter measurement could have caused the foulant layer to have a structural parameter during RO operation that was smaller than the thickness measured ex situ. 
The procedure described above was repeated for five sodium chloride concentrations, each with $200 \mathrm{mg} / \mathrm{L}$ of sodium alginate and $1 \mathrm{mM}$ of calcium chloride. The pore size and structural parameter for each is fit using the full model (Sec. 2) from the flux vs. glucose concentration data, and the average sodium and calcium ion concentrations in the cake layer are calculated. The osmotic pressure increase (CEOP) and hydraulic pressure drop through the gel are also calculated from the model and averaged between the three glucose concentrations to reveal which cause of flux decline dominates. We define the hydraulic loss ratio as the fraction of the change in hydraulic pressure $P$ minus osmotic pressure $\pi$ across the cake layer that is due to the difference in hydraulic pressure, $\left(\left[P_{c}-P_{m}\right] /\left[P_{c}-P_{m}+\pi_{m}-\pi_{c}\right]\right)$, where the subscript $c$ refers to the outer edge of the cake and the subscript $m$ refers to the cake-membrane interface. Although it seems that calcium and sodium concentrations independently influence alginate gel properties, we give pore size and hydraulic loss ratio in terms of sodium:calcium concentration ratio, noting that these values only apply when the calcium ion concentration is on the order of $1 \mathrm{mM}$.

The calculated pore diameters and hydraulic loss ratios are shown in Fig. A.9. The sodium:calcium ratio is reported as the spatial average ratio within the cake layer, as calculated from the layered model with calcium chloride, sodium chloride, and glucose concentration profiles superimposed, thus neglecting interactions between different solutes. Calculated pore diameters ranged from 4.5 to $20 \mathrm{~nm}$, which is comparable to the range of pore sizes reported for various alginate samples and measurement methods in literature (see Sec. 3.3). Pore size increases with increasing sodium:calcium concentration ratio. Hydraulic loss ratio drops from near one at low sodium:calcium ratio to $<5 \%$ as the sodium:calcium ratio approaches 400, showing that for high sodium concentrations, hydraulic pressure drop can be neglected. The drop in hydraulic loss ratio with increasing sodium concentration at fixed calcium concentration is the result of both increasing pore size, which leads to decreasing hydraulic pressure drop, and increasing feed osmotic pressure, which leads to increasing 


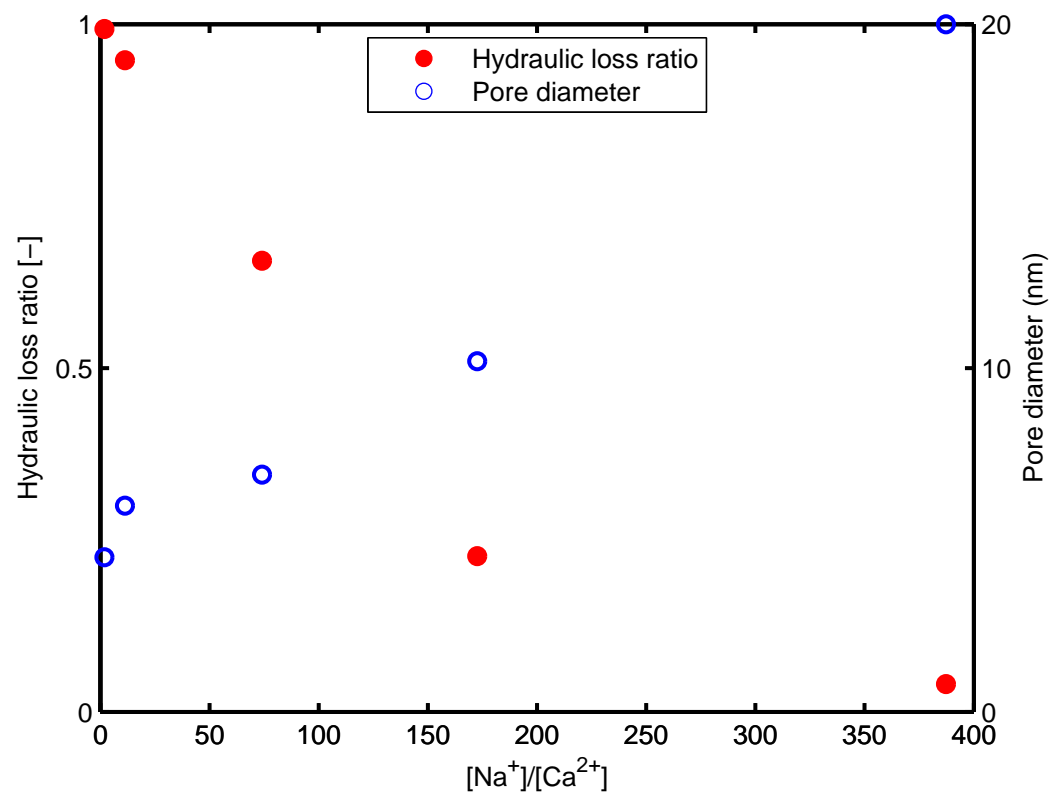

Figure A.9: Calculated hydraulic loss ratio (the fraction of flux decline caused by cake layer hydraulic resistance) and approximate pore diameter for a range of sodium:calcium concentration ratios with $1 \mathrm{mM}$ $\mathrm{CaCl}_{2}$ in the feed.

\section{CEOP.}

The cake structural parameter calculated from the model is highly sensitive to pore size when the pore size is low and flux decline is largely due to cake hydraulic drag. To avoid this regime until more precise pore measurements can be made, the minimum feed $\mathrm{NaCl}$ concentration considered in this study is $346 \mathrm{mM}(2 \%$ wt.) so that the hydraulic component of flux decline is less than approximately $10 \%$ and the resulting uncertainty in cake structural parameter is minimized. All other concentrations tested are above $400 \mathrm{mM}$, indicating a hydraulic loss ratio of $<5 \%$. Although no higher sodium:calcium ratios were tested, the hydraulic loss ratio is not expected to increase, and a pore size of $20 \mathrm{~nm}$ is assumed for all data analysis in the present study. However, future research on the pore size of organic foulants in various ionic environments will be useful for universally-applicable fouling analysis and imperative for the development of predictive modeling. 


\section{Appendix A.2. Membrane characterization}

Although membrane properties vary somewhat between coupons, it is not practical to characterize every membrane coupon individually. Salt permeation coefficients were calculated from the manufacturer's specifications, and other properties were characterized experimentally. To account for some variability between membrane samples, the water permeability of each RO coupon and the dispersivity of the FO coupon were fit from the initial flux before foulant cake formation.

\section{Appendix A.2.1. FO}

The FO membrane's salt permeation coefficient was calculated from its manufacturer specifications [29] and also measured experimentally.

Rejection, $R$, is specified as $99 \%$ and is defined in the membrane's specifications as:

$$
R=1-\frac{C_{f, 2} V_{f, 2}-C_{f, 1} V_{f, 1}}{\left(V_{f, 1}-V_{f, 2}\right) C_{d, a v e}}
$$

where $V_{f, 1}$ and $V_{f, 2}$ are the feed volume before and after the test, $C_{f, 1}$ and $C_{f, 2}$ are the feed salt concentration before and after the test, and $C_{d, a v e}$ is the average draw concentration during the test. Assuming values of $5 \times 10^{-5} \mathrm{~m} / \mathrm{s}$ for mass transfer coefficients on the feed and draw sides during the test, the salt permeation coefficient of the membrane was calculated from $R=99 \%$ using the foulant-free flux equations (Appendix C) as $B=5.32 \times 10^{-8} \mathrm{~m} / \mathrm{s}$.

To experimentally measure salt rejection, the system was run in FO mode for approximately three hours, during which time 159.9 g of liquid moved from the feed to the draw. Initial draw and feed conductivity were measured at $191.1 \mathrm{mS} / \mathrm{cm}$ and $59.5 \mu \mathrm{S} / \mathrm{cm}$, respectively. Final draw and feed conductivities were measured at $184.8 \mathrm{mS} / \mathrm{cm}$ and $169.5 \mu \mathrm{S} / \mathrm{cm}$, respectively. From the foulant-free FO model (Eqs. C.3 and C.4) with the measured flux, feed and draw salinities (averaged between initial and final values), and cross-flow velocities, a salt permeation coefficient of $B=1.37 \times 10^{-7} \mathrm{~m} / \mathrm{s}$ was fit from the data, corresponding to 
a salt rejection of $99.4 \%$. It is unsurprising that the measured salt rejection was higher than the specified salt rejection because the flux was much higher in our test (19.4 lmh vs. 9 lmh), resulting in significant ICP. However, despite our higher salt rejection, the salt permeation coefficient fit from our measurement was higher than that calculated from the specifications. A measurement made in a similarly-sized membrane module by Achilli et al. [42] with a CTA FO membrane from the same supplier found $B=1.11 \times 10^{-7} \mathrm{~m} / \mathrm{s}$, which is closer to our measurement. It seems to be common for coupon-sized membrane cells to have higher salt permeation, which is most likely due to increased salt permeability where the membranes are compressed by the sealing o-rings. Because salt transport through the bulk of the membrane (not the edges) affects the foulant cake salinity, we use the value $B=5.32 \times 10^{-8}$ $\mathrm{m} / \mathrm{s}$ that was calculated from the manufacturer's test with a larger membrane sample and, presumably, less significant edge effects.

Using the salt permeation coefficient calculated from the FO membrane specifications, the water permeability and the support layer structural parameter and dispersivity were fit from data collected from the FO unit over a wide range of feed and draw salinities. Using $\mathrm{NaCl}$ feed solutions and degassed $\mathrm{NaCl}$ draw solutions of various concentrations, the system was run with membranes in FO orientation (feed contacting active layer) for at least 30 minutes and until a stable flux was reached for each pair of feed and draw salinities. High cross-flow velocity $(22.2 \mathrm{~cm} / \mathrm{s})$ was used on the feed side to prevent fouling. Feed and draw conductivity were measured for each test, and the parameters of interest were fit from the data and the foulant-free matrix equations (Eqs. C.3 and C.4). The experimental data and corresponding calculated values based on the fitted membrane parameters are shown in Fig. A.10 along with the values that would be calculated using the fitted parameters of Achilli et al. [42] and neglecting dispersion.

The values attained from the fit of the experimental data were $A=1.9 \times 10^{-12} \mathrm{~m} / \mathrm{s}-\mathrm{Pa}$, $S_{S}=5.7 \times 10^{-4} \mathrm{~m}$, and $\alpha=1.65 \times 10^{-4} \mathrm{~m}$. The calculated permeability was similar to 


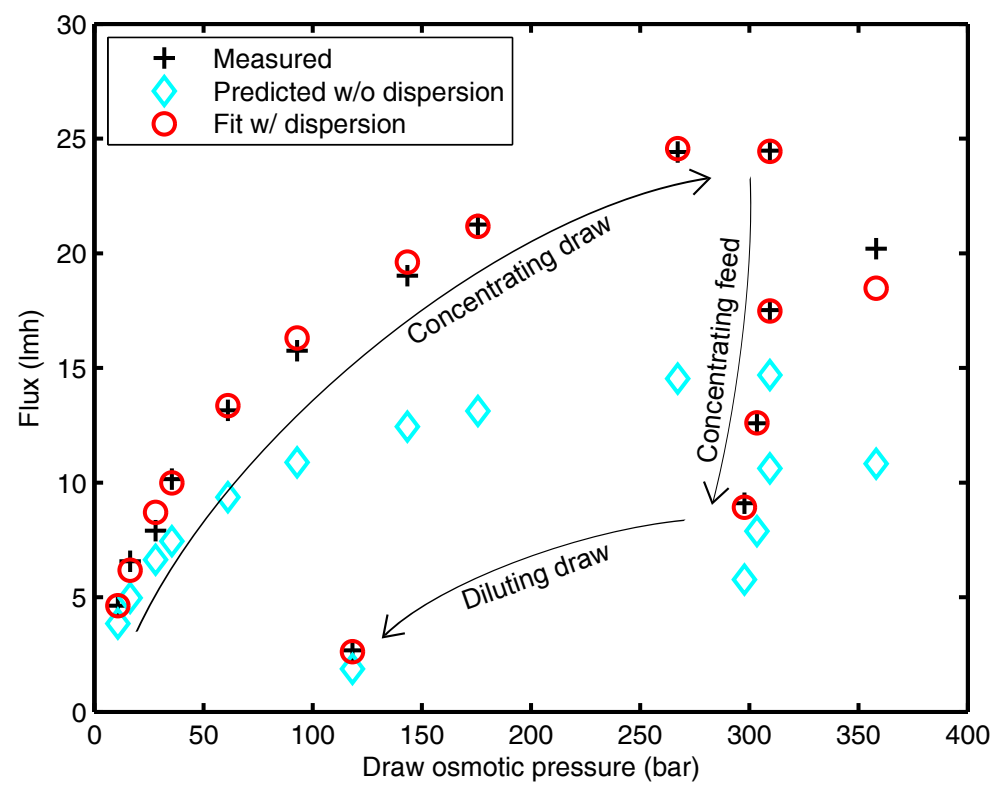

Figure A.10: Plot showing agreement between measured and predicted flux in FO based on a fit of permeability, structural parameter, and dispersivity across a range of feed and draw concentrations. Feed concentrations (not shown) ranged from 0-9\% wt. NaCl. Flux predictions for the same experimental conditions using the membrane properties calculated by Achilli et al. [42] without dispersion are given for comparison.

that measured by Achilli et al. [42] $\left(A=1.87 \times 10^{-12} \mathrm{~m} / \mathrm{s}-\mathrm{Pa}\right)$ in a pressurized pure water flux test with a membrane of the same type from the same manufacturer. The structural parameter fit by Achilli et al. was slightly greater than our value $\left(S_{S}=6.78 \times 10^{-4} \mathrm{~m}\right)$, but agrees in order of magnitude. Figure A.10 shows that with the membrane parameters fitted by Achilli et al. (with $\alpha=0$ because dispersion was not considered), the outcome is relatively well-predicted when the flux and draw osmotic pressure are low, but does not capture the behavior at high flux or high draw osmotic pressure. The fitted dispersion coefficient, as defined in Eq. 14, is within the range that would be expected for this membrane: using the the ratio $\tau_{S} / \epsilon_{S}=S_{S} / \delta_{S}=7.125$ (based on measured membrane thickness), Eq. 15 gives an estimate of $6.5 \mu \mathrm{m}$ for the product of pore diameter and inhomogeneity factor in the support layer, which is somewhat smaller than but comparable in order of magnitude to the 
pore sizes seen in micrographs [20] of the same type of FO membrane used here.

\section{Appendix A.2.2. RO}

Based on the measured feed conductivity and permeate mass and conductivity before and after eight hours of running $\mathrm{RO}$ with $3.5 \% \mathrm{NaCl}$ feed, the salt rejection of the membrane module was estimated as $99.55 \%$, which is close to the membrane's minimum rated salt rejection of $99.65 \%$ [43]. As with the FO membrane, the decrease in rejection is attributed to the small scale of the module and the rated rejection is used to calculate salt permeation coefficient. Using the foulant-free RO equations and the test conditions reported in the membrane data sheet [43], the salt permeation coefficient was determined to be $B=2.38$ $\times 10^{-8} \mathrm{~m} / \mathrm{s}$. Permeability, which varied between samples, was fit for each coupon from the flux before fouling.

\section{Appendix A.3. Mass transfer coefficients}

Convective mass transfer affects flux in both $\mathrm{FO}$ and $\mathrm{RO}$, but it is especially important to have a good estimate for the draw side mass transfer coefficient in FO because the high draw concentration results in a large concentration difference across the boundary layer (see Figs. 3 and 4). Mass transfer coefficients vary widely between studies, so for the purpose of analyzing the present experimental results, mass transfer coefficients were measured using the present experimental setup in an RO configuration. Flux was measured first with deionized water feed (no concentration polarization), and then with $3.5 \%$ wt. $\mathrm{NaCl}$ with the set of two fine spacers used in the draw channel in FO at two flow rates and with the coarse spacer used in the feel channels at one flow rate. The pulsation dampener typically used in the feed side of the apparatus was removed to simulate the pulsating flow that occurs in the draw channel.

The wetted area of the membrane is slightly larger than the channel area because of the offset O-ring. The O-ring meets the membrane $3.5 \mathrm{~mm}$ beyond the edge of the feed channel at the end of a gap less than $100 \mu \mathrm{m}$ thick. In the absence of concentration polarization 
(i.e., in the pure water RO test mentioned above), we assume that the membrane in the gap is active; however, with saline feeds, we assume that the concentration polarization within the gap is so severe that the water permeation in the gap is negligible.

Using the foulant-free model (Appendix C) and the measured fluxes, the mass transfer coefficients were calculated to be $1.121 \times 10^{-4}$ and $2.89 \times 10^{-5} \mathrm{~m} / \mathrm{s}$ for the twin fine spacers with feed velocities of 23.9 and $4.3 \mathrm{~cm} / \mathrm{s}$, respectively. The mass transfer coefficient with the single coarse spacer was measured as $3.61 \times 10^{-5} \mathrm{~m} / \mathrm{s}$ with a flow velocity of $8.3 \mathrm{~cm} / \mathrm{s}$. A correlation for long, spacer-filled channels by Koutsou et al. [44] gives mass transfer coefficients of a similar magnitude. In the absence of a correlation for developing, pulsating flow in channels with the present spacers, we assume a correlation of the form

$$
\mathrm{Sh}_{D_{h}} \propto\left[\left(D_{h} / l\right) \operatorname{Re}_{D_{h}} \mathrm{Sc}\right]^{n}
$$

and fit the exponent $n$ from the two flow velocities with the twin fine spacers as 0.79 . In Eq. A.2, $D_{h}$ is the channel hydraulic diameter, $\mathrm{Sh}_{D_{h}}$ is the Sherwood number, Sc is the Schmidt number, and $l$ is the channel length. For the present analysis of experimental data, we use the following correlations for mass transfer coefficient, where $v$ is the cross-flow velocity, which are based on the form of Eq. A.2 with values measured experimentally: Eq. A.3 for the twin fine spacers and Eq. A.4 for the coarse spacer.

$$
\begin{aligned}
& k_{\text {fine }}=1.121 \times 10^{-4}[\mathrm{~m} / \mathrm{s}] \times\left(\frac{v}{0.239[\mathrm{~m} / \mathrm{s}]}\right)^{0.79} \\
& k_{\text {coarse }}=3.61 \times 10^{-5}[\mathrm{~m} / \mathrm{s}] \times\left(\frac{v}{0.083[\mathrm{~m} / \mathrm{s}]}\right)^{0.79}
\end{aligned}
$$

There are several outstanding issues with the use of these empirical correlations in analyzing the present experiment. Although the mass transfer coefficients were measured in pulsating flow to simulate conditions in the draw channel, these correlations are also used in 
the feed channel, where the pulsation dampener significantly reduces flow oscillations. The direction of permeation velocity is opposite between the feed and draw channels, although the permeation velocity is very small compared to the cross-flow velocity $(\mu \mathrm{m} / \mathrm{s}$ rather than $\mathrm{cm} / \mathrm{s}$ ). The difference in viscosity between alginate-containing feed solutions and foulantfree feeds is not accounted for, nor is the difference in sodium chloride diffusion coefficient between different boundary layer concentrations. Further investigation of mass transfer coefficients in spacer-filled channels will be beneficial for fouling prediction, but it is beyond the scope of the present work.

\section{Appendix A.4. Accounting for time-varying solution concentrations}

Even in the absence of fouling, flux is expected to decline in this experiment because the feed and draw concentrations are not controlled. Instead, feed and draw concentrations are predicted over time using conservation of species based on changes in measured permeate/draw mass and assuming negligible salt permeation or evaporation.

Cake structural parameter is calculated from the theory in Sec. 2 based on the feed and, in FO, draw solution concentrations computed from the change in scale mass. Foulant-free flux is predicted based on the calculated feed and draw concentrations using the foulantfree matrix equations given in Appendix C. Flux decline is then calculated as the ratio of measured flux to predicted foulant-free flux.

\section{Appendix B. Modeling details}

\section{Appendix B.1. Concentration-dependence of membrane water permeability}

According to the solution-diffusion model, membrane water permeability scales with feed water concentration [13]. Therefore, to more accurately predict foulant accumulation from flux decline, we correct for the change in water permeability as the water concentration at 
the membrane, $C_{w, m}$, changes due to CEOP using Eq. B.1 for $\left.A\right|_{C_{m}}$ :

$$
\left.A\right|_{C_{m}}=\left.A\right|_{C_{m, 0}} \frac{C_{w, m}}{C_{w, m, 0}}=\left.A\right|_{C_{m, 0}} \frac{\left.\rho\right|_{C_{m}}\left(1-\left.s\right|_{C_{m}}\right)}{\left.\rho\right|_{C_{m, 0}}\left(1-\left.s\right|_{C_{m, 0}}\right)},
$$

where $C_{w, m, 0}$ and $C_{m, 0}$ are the water and salt concentrations present when permeability was evaluated and $s$ is the salt mass fraction. The dependence of permeability on water concentration results from the way permeability is defined: although flux is proportional to the gradient in solvent concentration within the membrane [13], permeability is defined as the ratio of flux to hydraulic--osmotic pressure difference outside the membrane. In Eq. B.1, any potential reduction in water concentration due to displacement by the foulant material is neglected in the current form of the model because of the high porosity of the alginate gels considered experimentally. However, the effect of nanostructured gels on water activity and membrane permeability could be a worthwhile subject for future investigation.

\section{Appendix B.2. Diffusion coefficients}

The $\mathrm{NaCl}$ diffusion coefficient itself varies about $10 \%$ across the range from dilute to saturated solutions [25], and the diffusion coefficient is generally higher on the draw side of the membrane. The variation in diffusion coefficient is taken into account by using roughly average values of diffusion coefficient in the support layer and foulant cake layer of $D_{S}=1.3673 \times 10^{-9} \mathrm{~m}^{2} / \mathrm{s}$ and $D_{C}=1.2939 \times 10^{-9} \mathrm{~m}^{2} / \mathrm{s}$, respectively. These values are based on the data of Vitagliano and Lyons [25] at $25{ }^{\circ} \mathrm{C}$ and corrected to $20{ }^{\circ} \mathrm{C}$ (the temperature maintained in our experiments) using the Stokes-Einstein relation.

\section{Appendix C. Layered model matrix equations}

The systems of equations presented herein for the layered transport models of FO and RO can be reduced to one equation each for the salt continuity and water flux, but the complexity of the salt flux equation increases with the number of layers. For the five-layer 
fouled FO model, we combine the equations for salt flux into a matrix equation that can be solved simultaneously with the corresponding equation for water flux in MATLAB. The matrix equation is created by subtracting the equation for salt flux through the active layer from each of the other salt flux equations. Some terms in the fouled equations are undefined when there is no foulant layer $\left(S_{C}=0\right)$, so equations for flux in the absence of fouling are also given here.

For FO with fouling, Eqs. C.1 and C.2 are solved simultaneously.

$$
\begin{aligned}
& {\left[\begin{array}{cccc}
\frac{-J_{w}}{\exp \left(J_{w} / k_{F}\right)-1} & -B & B & 0 \\
\frac{J_{w} \exp \left(J_{w} S_{C} / D_{C}\right)}{\exp \left(J_{w} S_{C} / D_{C}\right)-1} & \frac{-J_{w}}{\exp \left(J_{w} S_{C} / D_{C}\right)-1}-B & B & 0 \\
0 & -B & \frac{J_{w} \exp \left(J_{w} S_{S} / D_{S, \text { eff }}\right)}{\exp \left(J_{w} S_{S} / D_{S, \text { eff }}\right)-1}+B & \frac{-J_{w}}{\exp \left(J_{w} S_{S} / D_{S, \text { eff }}\right)-1} \\
0 & -B & B & \frac{J_{w} \exp \left(J_{w} / k_{D}\right)}{\exp \left(J_{w} / k_{D}\right)-1}
\end{array}\right]} \\
& \times\left[\begin{array}{c}
C_{c} \\
C_{m} \\
C_{s} \\
C_{b}
\end{array}\right]=\left[\begin{array}{c}
\frac{-J_{w} C_{f} \exp \left(J_{w} / k_{F}\right)}{\exp \left(J_{w} / k_{F}\right)-1} \\
0 \\
0 \\
\frac{J_{w} C_{d}}{\exp \left(J_{w} / k_{D}\right)-1}
\end{array}\right] \\
& J_{w}=\left.A\right|_{C_{m}}\left(\left.\pi\right|_{C_{s}}-\left.\pi\right|_{C_{m}}-\frac{32 \mu S_{C} J_{w}}{D_{h, C}^{2}}\right) ;
\end{aligned}
$$

Note that $D_{S, \text { eff }}$ is itself a function of $J_{w}$ (Eq. 14).

For FO without fouling, the equations to solve are Eqs. C.3 and C.4.

$$
\begin{gathered}
{\left[\begin{array}{ccc}
\frac{-J_{w}}{\exp \left(J_{w} / k_{F}\right)-1}-B & B & 0 \\
-B & \frac{J_{w} \exp \left(J_{w} S_{S} / D_{S, \text { eff }}\right)}{\exp \left(J_{w} S_{S} / D_{S, \text { eff }}\right)-1}+B & \frac{-J_{w}}{\exp \left(J_{w} S_{S} / D_{S, \text { eff }}\right)-1} \\
-B & B & \frac{J_{w} \exp \left(J_{w} / k_{D}\right)}{1-\exp \left(J_{w} / k_{D}\right)-1}
\end{array}\right] \times\left[\begin{array}{c}
C_{m} \\
C_{s} \\
C_{b}
\end{array}\right]=\left[\begin{array}{c}
\frac{-J_{w} C_{f} \exp \left(J_{w} / k_{F}\right)}{\exp \left(J_{w} / k_{F}\right)-1} \\
0 \\
\frac{J_{w} C_{d}}{\exp \left(J_{w} / k_{D}\right)-1}
\end{array}\right]} \\
\text { (C.3) } \\
J_{w}=\left.A\right|_{C_{m}}\left(\left.\pi\right|_{C_{s}}-\left.\pi\right|_{C_{m}}\right)
\end{gathered}
$$


For RO with fouling, Eqs. C.5 and C.6 are solved simultaneously.

$$
\begin{gathered}
{\left[\begin{array}{ccc}
\frac{-J_{w}}{\exp \left(J_{w} / k_{F}\right)-1} & -B & B \\
\frac{J_{w} \exp \left(J_{w} S_{C} / D_{C}\right.}{\exp \left(J_{w} S_{C} / D_{C}\right)-1} & \frac{-J_{w}}{\exp \left(J_{w} S_{C} / D_{C}\right)-1}-B & B \\
0 & -B & J_{w}+B
\end{array}\right] \times\left[\begin{array}{c}
C_{c} \\
C_{m} \\
C_{p}
\end{array}\right]=\left[\begin{array}{c}
\frac{-J_{w} C_{f} \exp \left(J_{w} / k_{F}\right)}{\exp \left(J_{w} / k_{F}\right)-1} \\
0 \\
0
\end{array}\right]} \\
J_{w}=\left.A\right|_{C_{m}}\left(P_{f}-\frac{32 \mu S_{C} J_{w}}{D_{h, C}^{2}}-\left[\left.\pi\right|_{C_{m}}-\left.\pi\right|_{C_{p}}\right]\right)
\end{gathered}
$$

For RO without fouling, Eqs. C.7 and C.8 can be used.

$$
\begin{gathered}
{\left[\begin{array}{cc}
\frac{-J_{w}}{\left(\exp \left(J_{w} / k_{F}\right)\right)-1}-B & B \\
-B & J_{w}+B
\end{array}\right] \times\left[\begin{array}{c}
C_{m} \\
C_{p}
\end{array}\right]=\left[\begin{array}{c}
\frac{-J_{w} C_{f} \exp \left(J_{w} / k_{F}\right)}{\exp \left(J_{w} / k_{F}\right)-1} \\
0
\end{array}\right]} \\
J_{w}=\left.A\right|_{C_{m}}\left(P_{f}-\left[\left.\pi\right|_{C_{m}}-\left.\pi\right|_{C_{p}}\right]\right)
\end{gathered}
$$

Once the equations for water flux and concentrations have been solved simultaneously, salt flux (if desired) can be calculated from one of the algebraic equations for salt flux in Sec. 2, e.g., Eq. 9 for FO or Eq. 17 for RO.

\section{Appendix D. Concentration and pressure profile equations}

The layered transport model allows for the evaluation of concentration and pressure profiles throughout the various layers in fouled FO and $\mathrm{RO}$. In this section, we present equations for concentration and pressure profiles based on interface concentrations and pressures, which must be calculated first using the equations in Sec. 2 or Appendix C. The equations given here are based on distance, $x$, which increases in the direction of water flow and originates at the feed side of the membrane active layer.

To compute the concentration and pressure profiles, the thickness of the various layers must be known. The thickness of the feed and draw boundary layers can be computed from 
the mass transfer coefficients as $\delta_{F}=D_{C} / k_{F}$ and $\delta_{D}=D_{S} / k_{D}$, respectively, assuming the average diffusion coefficients in the porous layers are similar to those in the neighboring boundary layers. The membrane active layer, which is around $\delta_{A}=200 \mathrm{~nm}$ thick in RO membranes [45], is very thin compared to the other layers. The support layer thickness of a membrane can be measured with calipers or a micrometer, assuming that the contribution of the active layer to the thickness is negligible. The RO and FO membranes used in the present experiments were measured with calipers to be 150 and $80 \mu \mathrm{m}$ thick, respectively. The thickness of the foulant cake can be measured experimentally or estimated from the calculated cake structural parameter and values of porosity and tortuosity taken from the literature (e.g., [16]), but the high porosity and correspondingly low tortuosity of alginate gels suggest that $\delta_{C}=S_{C}$ is a reasonable first approximation in many cases.

For FO, the concentration and (gauge) pressure profiles are piecewise equations (Eqs. D.1 and D.2) based on the models for each layer as shown in Fig. 1. The equations for the cake layer $\left(-\delta_{C} \leq x<0\right)$ assume that the pore size, tortuosity, and porosity are uniform within the cake layer, but future work may relax this assumption. The sorption coefficient, $K$, that appears in the active layer is a result of the solution-diffusion model [13].

$$
C(x)= \begin{cases}C_{f} & x \leq-\left(\delta_{F}+\delta_{C}\right) \\ C_{f}+\left(C_{c}-C_{f}\right) \frac{\exp \left(\left[x+\delta_{F}+\delta_{C}\right] J_{w} / D_{C}\right)-1}{\exp \left(\delta_{F} J_{w} / D_{C}\right)-1} & -\left(\delta_{F}+\delta_{C}\right) \leq x \leq-\delta_{C} \\ C_{c}+\left(C_{m}-C_{c}\right) \frac{\exp \left(\left[x+\delta_{C}\right] J_{w} S_{C} /\left[D_{C} \delta_{C}\right]\right)-1}{\exp \left(S_{C} J_{w} / D_{C}\right)-1} & -\delta_{C} \leq x<0 \\ K\left(C_{m}+\frac{x}{\delta_{A}}\left[C_{s}-C_{m}\right]\right) & 0<x<\delta_{A} \\ C_{s}+\left(C_{b}-C_{s}\right) \frac{\exp \left(\left[x-\delta_{A}\right] J_{w} S_{S} /\left[D_{S, \mathrm{eff}} \delta_{S}\right]\right)-1}{\exp \left(S_{S} J_{w} / D_{S, \mathrm{eff}}\right)-1} & \delta_{A}<x \leq \delta_{A}+\delta_{S} \\ C_{b}+\left(C_{d}-C_{b}\right) \frac{\exp \left(\left[x-\delta_{A}-\delta_{S}\right] J_{w} / D_{S}\right)-1}{\exp \left(\delta_{D} J_{w} / D_{S}\right)-1} & \delta_{A}+\delta_{S} \leq x \leq \delta_{A}+\delta_{S}+\delta_{D} \\ C_{d} & x \geq \delta_{A}+\delta_{S}+\delta_{D}\end{cases}
$$




$$
P(x)= \begin{cases}0 & x \leq-\delta_{C} \\ -\frac{x+\delta_{C}}{\delta_{C}} \frac{32 \mu S_{C} J_{w}}{D_{h, C}^{2}} & -\delta_{C} \leq x \leq 0 \\ -\frac{32 \mu S_{C} J_{w}}{D_{h, C}^{2}} & 0 \leq x<\delta_{A} \\ 0 & x>\delta_{A}\end{cases}
$$

The RO concentration and (gauge) pressure profiles are given in Eqs. D.3 and D.4.

$$
\begin{gathered}
C(x)= \begin{cases}C_{f} & x \leq-\left(\delta_{F}+\delta_{C}\right) \\
C_{f}+\left(C_{c}-C_{f}\right) \frac{\exp \left(\left[x+\delta_{F}+\delta_{C}\right] J_{w} / D_{C}\right)-1}{\exp \left(\delta_{F} J_{w} / D_{C}\right)-1} & -\left(\delta_{F}+\delta_{C}\right) \leq x \leq-\delta_{C} \\
C_{c}+\left(C_{m}-C_{c}\right) \frac{\exp \left(\left[x+\delta_{C}\right] J_{w} S_{C} /\left[D_{C} \delta_{C}\right]\right)-1}{\exp \left(S_{C} J_{w} / D_{C}\right)-1} & -\delta_{C} \leq x<0 \\
K\left(C_{m}-\frac{x}{\delta_{A}}\left[C_{m}-C_{p}\right]\right) & 0<x<\delta_{A} \\
C_{p} & x>\delta_{A}\end{cases} \\
P(x)= \begin{cases}P_{f} & x \leq-\delta_{C} \\
P_{f}-\frac{x+\delta_{C}}{\delta_{C}} \frac{32 \mu S_{C} J_{w}}{D_{h, C}^{2}} & -\delta_{C} \leq x \leq 0 \\
P_{f}-\frac{32 \mu S_{C} J_{w}}{D_{h, C}^{2}} & 0 \leq x<\delta_{A} \\
0 & x>\delta_{A}\end{cases}
\end{gathered}
$$

\section{Appendix E. Uncertainty analysis}

Flux decline and cake structural parameter are both calculated from measured flux, which itself has several sources of uncertainty. Here, we estimate the $95 \%$ confidence interval for our flux decline and cake structural parameter measurements using the propagation of uncertainty method.

Flux uncertainty arises from high-frequency fluctuations, low-frequency thermal fluctuations, and possible changes in membrane properties over time. In the present experiments, measured flux varies at a high frequency with an amplitude of about $0.10 \mathrm{lmh}$ for RO and $0.19 \mathrm{lmh}$ for FO (the standard deviation of flux measurements every 5 minutes for one hour 
at the end of a test when the flux decline was very slow). Based on the statistical uncertainty method with 12 measurements, this would indicate a $95 \%$ confidence interval of $\pm 0.22 \mathrm{lmh}$ in $\mathrm{RO} \pm 0.41 \mathrm{lmh}$ in $\mathrm{FO}$ for these fluctuations, which seem to occur due to the low resolution of the digital scale compared to the mass flow into it during the 15 minute flux measurement interval. The larger variation in FO may be partly explained by the connection of the draw volume on the scale to the draw loop, whose volume fluctuates slightly due to the motions of the diaphragm pump. Flux also seems to vary over a period of hours, which is likely due to fluctuations in temperature. The temperature control system maintains the temperature between 20 and $21^{\circ} \mathrm{C}$. According to the model of Jin et al. [46] for RO membrane permeability temperature dependence, this difference should result in a $\pm 1.2 \%$ permeability variation. Neglecting the effects of other layers on flux, the solution-diffusion model [13] would thus predict a $\pm 1.2 \%$ variability in flux due to imperfect temperature control. Membrane properties could also change due to incomplete conditioning before beginning experiments, and to account for this, an uncertainty of $\pm 1 \%$ of flux is assumed. Propagation of uncertainty leads to a $95 \%$ confidence interval of approximately $2.5 \%$ of flux in RO and $4.0 \%$ of flux in FO based on the initial flux range of the present experiments.

Flux decline is the ratio of measured flux to predicted foulant-free flux. The latter is based on permeability measured from the initial flux, so the flux uncertainty applies to both measurements. The propagation of uncertainty method then dictates that the $95 \%$ confidence interval in flux decline is \pm 3.6 and \pm 5.7 percentage points in $\mathrm{RO}$ and $\mathrm{FO}$, respectively.

Structural parameter uncertainty is based on uncertainties in flux and initial flux plus uncertainty in the assumption of $20 \mathrm{~nm}$ foulant pore size. The error in $S_{C}$ due to the assumed $20 \mathrm{~nm}$ pore size is estimated to be $5 \%$ in the lowest salinity $(2 \% \mathrm{NaCl}) \mathrm{RO}$ trial, and $2 \%$ for the higher salinity trials, where the effect of cake hydraulic resistance becomes negligible (see Fig. A.9). The change in calculated structural parameter based on a small change 
in measured flux varies depending on many variables, but to provide an estimate of the uncertainty for all trials, this derivative is calculated numerically for representative FO and $\mathrm{RO}$ cases near the end of the fouling run $\left(S_{C} \approx 100 \mu \mathrm{m}\right.$ for $\mathrm{RO}$ and $S_{C} \approx 200 \mu \mathrm{m}$ for $\left.\mathrm{FO}\right)$ as $1.64 \times 10^{-5} \mathrm{~m} / \mathrm{lmh}$ for $\mathrm{RO}$ and $3.14 \times 10^{-5} \mathrm{~m} / \mathrm{lmh}$ for FO. The greater change in cake structural parameter needed to change the flux by a given amount in FO is primarily due to the effect of internal concentration polarization. Propagation of uncertainty considering uncertainties in flux, initial flux, and pore size leads to the following $95 \%$ confidence intervals in cake structural parameter: $\pm 40 \mu \mathrm{m}$ for the FO test, $\pm 14 \mu \mathrm{m}$ for $\mathrm{RO}$ with $2 \% \mathrm{NaCl}$, and $\pm 13 \mu \mathrm{m}$ for the other (higher-salinity) RO tests.

There may be additional uncertainty in structural parameter due to uncertainty in dispersivity in the support layer and mass transfer coefficients in the feed and draw channels. Further research is needed in these areas. 
Flux decline quantifies

system response to fouling

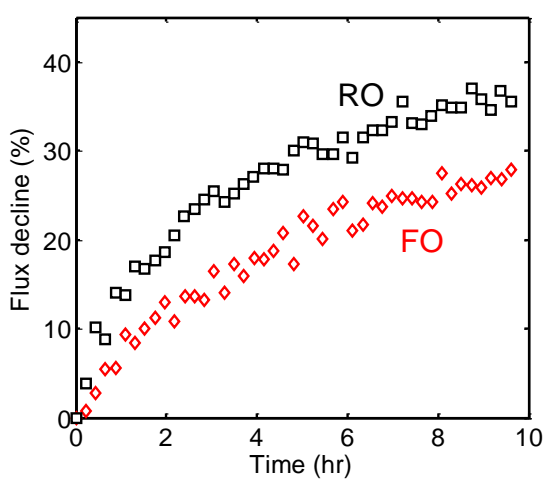

Structural parameter quantifies effective thickness of foulant

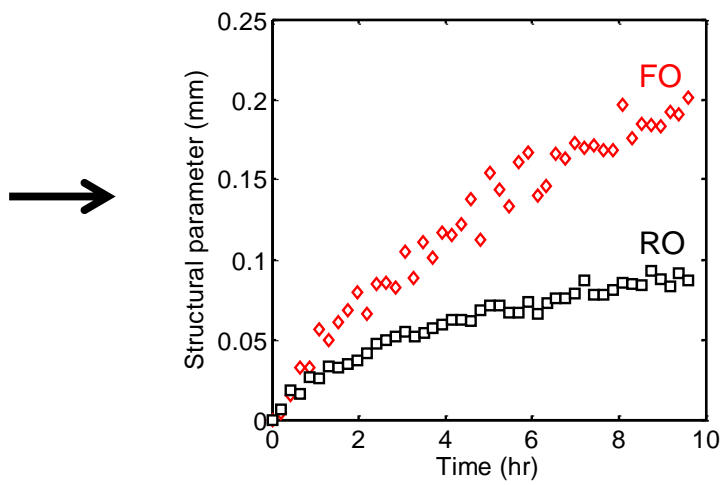

